\title{
Épistémologie de la turcologie
}

\section{Emmanuel Szurek}

\author{
(2) OpenEdition \\ Journals \\ Édition électronique \\ URL : http://journals.openedition.org/ejts/5524 \\ DOI : $10.4000 /$ ejts. 5524 \\ ISSN : $1773-0546$ \\ Éditeur \\ EJTS
}

Référence électronique

Emmanuel Szurek, «Épistémologie de la turcologie », European Journal of Turkish Studies [En ligne], 24 | 2017, mis en ligne le 08 novembre 2017, consulté le 10 décembre 2020. URL : http://

journals.openedition.org/ejts/5524; DOI : https://doi.org/10.4000/ejts.5524

Ce document a été généré automatiquement le 10 décembre 2020.

(c) Some rights reserved / Creative Commons license 


\title{
Épistémologie de la turcologie
}

\author{
Emmanuel Szurek
}

À Étienne Copeaux, inspirateur de la première heure.

1 Pour les « gens ordinaires» (ceux qui tiennent les notions d'astrologie, d'œnologie ou même de sismologie pour des denrées banalisées) le mot même est source d'étonnement. Sa simple prononciation suscite souvent, auprès du grand public, des manifestations d'incrédulité, parfois matinées d'amusement ( - La turcologie? vraiment? ça existe?»). Quelquefois, chez les universitaires - en particulier du côté des études littéraires et des cultural studies -, c'est plutôt le malaise qui prime : comment peut-on persister à utiliser un terme qui connote si manifestement la violence épistémique que les Européens ont exercée sur le reste du monde ? Quant aux « initiés », il y a ceux qui assument le mot, ceux qui l'utilisent sans trop y penser, et enfin ceux qui semblent s'en servir à regret en le flanquant de guillemets ou de modalisateurs divers, voire en lui préférant la catégorie d'études turques, peut-être jugée plus conforme à la bienséance contemporaine en sciences humaines et sociales ${ }^{1}$.

«Turcologie», «études turques»: de quoi parle-t-on au juste? En effet il n'est pas certain, que la turcologie soit autre chose qu'un mot. Les contributions de ce dossier le démontrent amplement : le terme désigne des pratiques à ce point diversifiées, dans le temps et dans l'espace, voire d'un individu à l'autre, qu'il est douteux qu'on puisse les référer à une architecture épistémologique commune. À l'inverse, une multiplicité de notions (turcologie, études turques, "science de la turcité », " étude de la Turquie »), plus ou moins traduisibles d'un pays et d'une langue à l'autre, sont disponibles sur le marché linguistique international. Dans l'usage académique elles désignent un « domaine », une « aire » ou un « champ d'études »: autant de métaphores spatialistes (et relativement tautologiques) qui permettent de lui imputer le statut de savoir, de savoir-faire ou de spécialité sans pour autant nécessairement lui reconnaître la consistance théorique d'une discipline ${ }^{2}$.

On arguera à bon droit qu'un tel flottement à la fois terminologique et épistémologique est le lot de bien des area studies (sinologie, iranologie, slavistique, French \& Italian studies etc.), dont le propre est de greffer sur un nom collectif (lui-même identifié selon 
des considérations ethniques, politiques, linguistiques ou confessionnelles) des répertoires de connaissance issus d'une pluralité de sciences humaines et sociales ${ }^{3}$. Pourtant ni le procès passablement éculé de l'orientalisme ni la remise en cause plus récente des aires culturelles n'épuisent la critique interne, à la fois historique et philologique, du mot si ce n'est de la chose turcologique. Or c'est peu dire que cette introspection est lacunaire: " il semble que la majorité des représentants de la turcologie - écrit Jens-Peter Laut, l'un des rares praticiens contemporains à s'être penché sur le problème de la définition - se soient résignés à travailler dans une discipline (Disziplin) dont les fondamentaux n'ont pas été clairement définis » (Laut $2013: 15)$.

4 Il reste que, même lâchement circonscrites, les études turques demeurent ici et là à travers le monde un principe concret d'organisation de la vie académique (administration de la recherche, cursus, financements, publications et recrutements universitaires). Si bien que, fût-ce sans échine intellectuelle, elles nous " en imposent " par mille trivialités quotidiennes, tant pratiques que cognitives, à commencer par leur nom qui est aussi celui de maints revues, départements et congrès internationaux. Ajoutons que si les turcologues de convention (expression qui peut s'entendre au sens propre aussi bien que figurét) sont sans doute les plus nombreux aujourd'hui, il existe encore, comme on le verra, des turcologues de conviction, c'est-à-dire des praticiens inspirés pour qui la chose n'est pas qu'un label mais une spécialité fondée en raison, justifiable d'états de l'art et de généalogies : autant d'opérations susceptibles de revêtir - ou de revendiquer pour le moins - une dimension prescriptive.

On entrevoit dès lors dans quelle direction orienter la réflexion : plutôt que de chercher l'improbable vérité de la turcologie, interrogeons celle des turcologues. Et si la turcologie n'est autre chose qu'un mot, demandons ce qu'on veut bien lui faire dire. Le premier temps de cet essai sera donc philologique. On y croisera des préoccupations théoriques (de quelles définitions dispose-t-on?), lexicologiques (comment le mot se traduit-il d'une langue à l'autre?) et pragmatiques (quels en sont l'usage et les variations sémantiques?). Nous verrons qu'une analyse de fond sur les mots de la turcologie ne peut faire l'économie d'une réflexion particulière sur le vocable turc luimême, dans ses déclinaisons historiques aussi bien que géographiques. Le second moment sera historiographique: que disons-nous de la turcologie lorsque nous entreprenons d'en raconter l'histoire? Différentes mises en récit seront examinées qui inscrivent les études turques dans autant de généalogies et d'historicités possibles. Les décrire phénoménologiquement, sans préjuger de l'autorité des références convoquées (de Wikipédia au Collège de France), ni de la validité intrinsèque des énoncés considérés, permettra d'esquisser, fût-ce de façon fragmentaire, quelque chose comme une tradition. L'objectif, en définitive, est de mieux traquer le corpus d' "évidences " (et de confusions) avec lesquelles les turcologues du XX $\mathrm{XX}^{\mathrm{e}}$ siècle - et peut-être encore ceux du temps présent - ont été conduits à se bricoler une identité intellectuelle.

\section{Philologie de la turcologie}

6 Voyage en Turcologie : première partie. On se demandera tout d'abord à quel point il faut prendre au sérieux les enjeux de classification inhérents à la multiplicité des labels qui servent à la désigner ("La "turcologie" contre les "études turques" ?»). Dans un second temps on se débattra avec une distinction relativement ésotérique pour le 
grand public et pourtant souvent considérée comme un marqueur de scientificité parmi les initiés : celle qu'on opère en français (et dans quelques autres langues) entre turc et turk. Enfin on se demandera comment interpréter le fait que cette distinction n'existe pas en turc de Turquie (voir infra "Turc ou Turk? Ce que la Turquie fait à la turcologie »).

\section{La « turcologie » contre les « études turques»?}

7 On a vu dans l'introduction de ce dossier que la turcologie n'admet pas de système théorique. Idéal-typiquement, la chose oscille entre deux registres de déchiffrement du monde qui conduisent à des régimes de compréhension du "fait turc» ou de la " turcité » différents, voire opposés. Soit la turcologie se fonde sur une aperception métalinguistique et fait droit à une certaine forme d'universalisme (cf. la formule de Louis Bazin rendant hommage à Robert Mantran : «Rien de turc ne lui est étranger »; Bazin 1988: 50), soit elle se fonde sur une lecture ethnographique, voire ethnologique (« La turcologie est une branche de la science qui traite des Turks») (Róna-Tas 1991 : 9). De cette lecture on glisse alors facilement vers l'ethnicisme, c'est-à-dire vers une vision du monde gouvernée par le sens et la quête de l'hérédité (races, peuples, ethnies).

En va-t-il autrement de ce que l'on désigne sous le label plus contemporain des « études turques »? Y a-t-il seulement matière à distinction ? En première approche, on serait tenté de répondre par l'affirmative, la turcologie relevant idéal-typiquement du « vieil » orientalisme ethnophilologique, quand les études turques connoteraient un tropisme prononcé pour les sciences sociales. En outre, le mot «turcologie » semblerait plutôt sur le déclin. Après avoir connu le sommet de sa carrière dans la seconde moitié du $\mathrm{XX}^{\mathrm{e}}$ siècle $^{5}$, il serait devenu ringard ${ }^{6}$. À l'inverse, le label études turques continue de justifier l'existence d'une multitude d'institutions universitaires à travers le monde. Par-delà ces considérations préliminaires, c'est un partage géographique qui se dessine ici : alors que la turcologie implique un imaginaire volontiers comparatiste indexé sur l'ensemble du monde turcique, les études turques semblent désigner des recherches centrées sur la seule Turquie. C'est dans cette perspective qu'il faut resituer les efforts du professeur Jens-Peter Laut de l'université de Gottingen pour restaurer la turcologie dans ses droits historiques:

[...] il faut veiller à ne pas trop vite mettre le turc de Turquie, et avec lui la Turquie dans ses différentes facettes contemporaines, au premier plan de la turcologie universitaire. Plus concrètement : bien que des considérations sur l'adhésion de la Turquie à l'UE, sur sa politique kurde, sur les migrations, les problèmes urbains à Istanbul ou encore sur ce qu'il est convenu d'appeler la ré-islamisation du pays ne devraient nullement être exclues ou ignorées par elle, mon sentiment personnel est que ces questions ne devraient pas constituer son cœur d'activité. Les politologues, sociologues, ethnologues, les spécialistes des religions et aussi les journalistes, du moins ceux ayant une bonne connaissance du turc, leur donnent aisément le change sur ce terrain-là.

[...] il nous incombe de tirer le meilleur parti de la principale ressource de notre science (Wissenschaft), qui est la langue et son étude philologico-linguistique. Lui sont bien sûr liées l'évaluation critique des sources ainsi que d'autres méthodes dont la mise en œuvre permet d'aboutir à des résultats solides en histoire, en linguistique, dans le domaine de l'analyse littéraire ou culturelle tout comme en science des religions. À leur tour, des disciplines telles que la science politique, la sociologie et l'histoire peuvent et doivent s'appuyer sur ces résultats. Mais, voyezvous, la turcologie doit proposer quelque chose qu'elle est seule à pouvoir offrir, 
faute de quoi elle court le danger d'être ravalée au rang de science auxiliaire des disciplines précitées ainsi que d'autres (et encore une qui ne soit pas trop prise au sérieux), considérée uniquement pour répondre à des sollicitations du type (et j'en ai fait l'expérience) : « Veuillez me traduire le dernier discours du Premier ministre Erdoğan » ou encore «Au fait, pourquoi les Ouzbeks ont-il été choisir le terrible Timour comme ancêtre national ?» (Laut 2013 : 19-20).

La démarche, on l'aura compris, est normative et programmatique plutôt que descriptive. L'auteur plaide pour une " dé-turquisation » et une "re-philologisation » de la turcologie: pour éviter l'impérialisme des sciences sociales (si ce n'est même camérales), et se prémunir contre le risque de dissolution du domaine, la seule solution est de remettre la Türkeikunde («étude de la Turquie ») à sa place : celle d'une belle province, certes, mais jamais qu'une subdivision de la Gesamtturkologie («turcologie totale $»^{7}$ ).

Le départage qui se dessine ici se reflète en miroir dans la façon dont la revue anglophone Turkish Studies, qui est abritée par la maison d'édition britannique Taylor \& Francis ${ }^{8}$, rend compte d'elle-même en sa rubrique "Objectifs et champs de vision " 9 , laquelle fut probablement rédigée au moment de la fondation de la revue, en 2000 - le propos n'aurait sans doute pas été exactement le même s'il avait été écrit de nos jours) :

La Turquie est un pays dont l'importance croît considérablement dans les affaires internationales. État démocratique qui connaît un développement accéléré grâce à une forte économie, une société complexe, un système partisan dynamique et de puissantes forces armées, la Turquie joue un rôle de plus en plus stratégique en Europe, au Moyen-Orient et au Caucase.

Au vu de l'importance du pays et de l'intérêt considérable que représente l'étude de son histoire, de sa vie politique et de sa politique étrangère, Turkish Studies se veut un forum de discussion scientifique sur ces sujets et d'autres.

11 L'exposé de la " politique éditoriale » de l'European Journal of Turkish Studies (2004) laisse un sentiment approchant. Bien que la revue publie des études centrées sur d'autres espaces, il y est question de relayer auprès du public francophone « les travaux menés à bien en Turquie » comme d'assurer aux articles publiés dans la revue "une visibilité au-delà des seuls spécialistes de la Turquie $»^{10}$. On observe en somme une sorte de chiasme épistémologique : tout se passe comme si, portées par la vocation instrumentale des area studies et travaillées au corps par les «tournants » théoriques successifs des cinquante dernières années, ce qu'on appelle "études turques » était voué à tutoyer l'ensemble des sciences sociales, mais à conserver la seule Turquie comme principal point d'ancrage empirique (quitte à s'autoriser des comparaisons vers l'extérieur), quand la «turcologie » resterait le gardien de l'ethno-philologie ancestrale, des Balkans à l'Asie centrale ${ }^{11}$.

12 Il reste que cette répartition géographico-disciplinaire est récente et des plus contingentes. Au siècle dernier, la distinction n'était guère pertinente. L'historien Franz Babinger nous en offre un premier exemple, parmi beaucoup d'autres. Tout juste revenu du front d'Orient (et après s'être associé à la répression de la République socialiste des conseils de Bavière), le jeune orientaliste publie en 1919 une enquête d'une trentaine de pages intitulée « Die türkischen Studien in Europa bis zum Auftreten Josef von Hammer-Purgstall ». Il s'agit en réalité d'une synthèse des savoirs produits sur les Ottomans par les Européens (en particulier ceux de langue allemande) du XV au $\mathrm{XVIII}^{\mathrm{e}}$ siècle ${ }^{12}$. Le texte se conclut par un hommage au drogman autrichien Joseph von Hammer-Purgstall. En l'auteur de l'Histoire de l'Empire ottoman (1827-1835) le professeur bavarois décèle le véritable fondateur de son domaine : 
Dépeindre l'influence de cet homme sur les études turques, auxquelles il a donné une direction toute nouvelle, excède le projet de cette esquisse. Josef v. Hammer est entré en scène au moment même où les sciences orientalistes connaissaient un essor immense et, là est l'essentiel, au moment même où la « turcologie » se taillait une province autonome dans l'immense empire des études orientales (Babinger $\left.1919: 129^{13}\right)$.

Le royaume des türkischen Studien, telles que les comprend Babinger en 1919, c'est l'Empire ottoman. Quant à la Turkologie, elle désigne au mieux une étape ultérieure sur la voie de leur « autonomisation » vis-à-vis de l'Orientalistik. Le contraste est saisissant entre, d'une part, l'ottomanocentrisme de l'universitaire munichois, qui semble ici se désintéresser des Turcs d'Asie centrale et des études menées en Europe à leur le sujet (dont l'histoire remonte pourtant à la fin du XVII ${ }^{e}$ siècle ${ }^{14}$ ), et, d'autre part, le tropisme de plus en plus prononcé de ses contemporains berlinois (Wilhelm Bang, Friedrich W. K. Müller, Albert von Le Coq, Joseph Marquart) aussi bien que pétersbourgeois (Wilhelm Radloff, Vassili Barthold, Alexander Samoïlovitch) vis-à-vis de la Sibérie et du Turkestan oriental.

14 Trente ans plus tard et quelques centaines de kilomètres plus à l'ouest, voici une affiche des conférences du Centre d'études turques de la Sorbonne pour l'année $1953^{15}$. On y trouve pêle-mêle, cette fois-ci, des interventions traitant de toutes les époques et de tous les mondes (de la Sibérie à l'Asie mineure, des Turcs célestes à ceux du temps présent) et procédant de toutes les méthodes et de toutes les disciplines: de l'ethnologie (Pertev Boratav) à l'ethnohistoire (Louis Bazin), de la paléographie (Irène Mélikoff) à la phonologie (Johannes Benzing), de l'histoire de l'art (Ali Toptchibachy) à celle des religions (Jean-Paul Roux), en passant par les études ottomanes (Mehmed Izeddin) - autrement désignée par le vocable d'«osmanologie » sous la plume de Louis Bazin (Bazin 1958: 116). En résumé, pour cette époque lointaine que fut le XXe siècle, on chercherait vainement à faire la part des choses. "Turcologie ", "études turques ", « études ottomanes » : tout cela coexistait dans une harmonieuse confusion. 


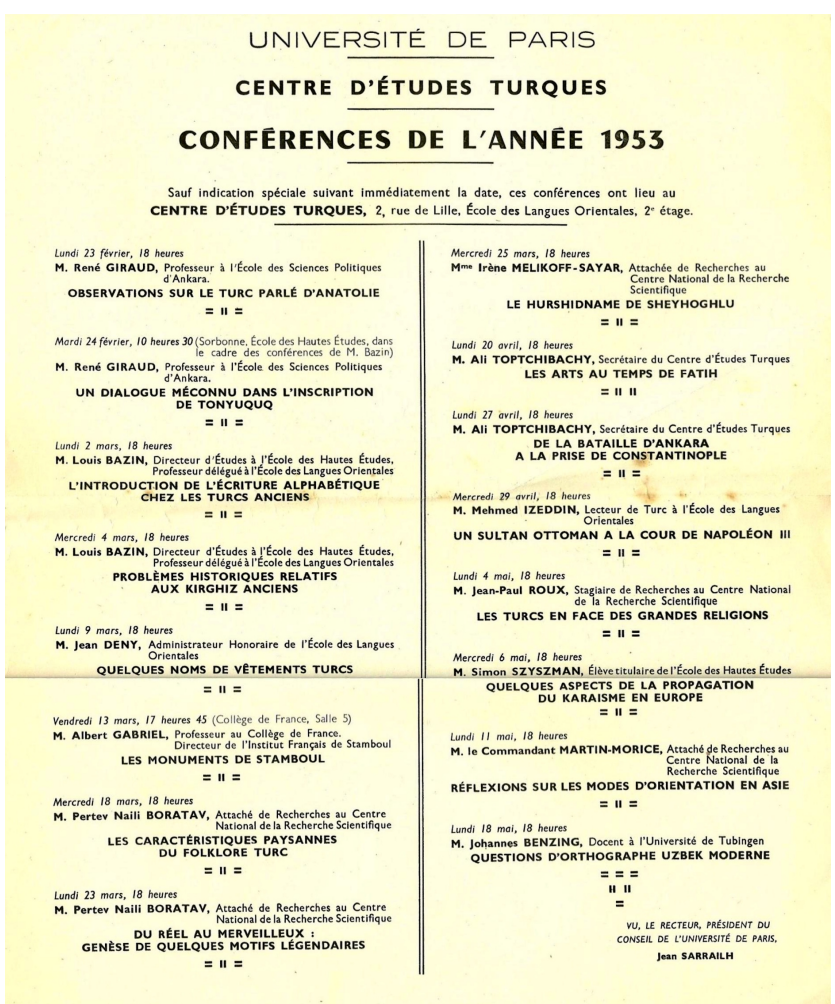

Fig. 1. Programme des conférences du Centre d'études turques de la Sorbonne (année 1953-1954). Archives privées de Jean Deny (EHESS, Paris).

15 Il n'est pas certain que le brouillard se soit totalement dissipé de nos jours. Il suffit en effet de se déplacer sur le terrain turcophone pour saisir tout ce que la nuance entre «turcologie » et "études turques» a d'illusoire si ce n'est d'amphigourique. Tout d'abord, en turc, la notion d'études turques, au sens d'études sur la Turquie, n'existe pas. Un Turc travaillant sur son pays n'est évidemment pas turcologue mais sociologue, anthropologue, littéraire ou historien ${ }^{16}$. Quant à la notion de Türkoloji, il faut compter avec les nombreux synonymes qui, en turc, lui font cortège (Türkiyat, Türkbilig, Türkbilimi, Türklük bilimi, Türklük Araştırmaları etc.). Pour prendre un exemple concret, rappelons que la faculté de lettres et d'histoire-géographie de l'université d'Ankara publie deux organes dont les préoccupations se recouvrent largement : l'une se nomme «revue de turcologie» (Türkoloji Dergisi), l'autre «revue d'études modernes sur la turcité» (Modern Türklük Araştırmaları Dergisi / Journal of Modern Turkish Studies). La première a été fondée en 1964, la seconde en 2004 : tout juste admettra-t-on que ce que l'on appelait «turcologie» voici un demi-siècle se trouve plus volontiers nommé "études turques» de nos jours. Et que ce que l'on entend par Türklük Araştırmaları ("recherches sur la turcité») en turc d'aujourd'hui trouve son équivalent dans le Turkish Studies de langue anglaise ${ }^{17}$.

Voilà de quoi décourager définitivement toute rigidité taxinomique. Sans doute de moins en moins nombreux sont les chercheurs qui songeraient à se revendiquer de la "vieille" turcologie. Mais il nous semble que répudier cette dernière pour mieux se prévaloir des «modernes » études turques, fût-ce par convention, c'est s'acquitter à trop bon compte de ce qui, par-delà leur différenciation disciplinaire et géographique, résiste, pour le meilleur ou pour le pire, de celle-ci dans celles-là. Qu'on se le dise : nous n'en n'avons pas fini avec la turcologie. 


\section{Turc ou Turk ? Ce que la Turquie fait à la turcologie} laissée de côté jusqu'ici. L'usage s'est en effet établi en français académique ainsi que dans quelques autres langues d'opérer une distinction entre turc et turk ${ }^{18}$. Turc est utilisé stricto sensu pour désigner la langue officielle de l'État établi vers 1920 sous le nom de Turquie et plus généralement ce qui ressortit à la société turque contemporaine. Turk (ou turcique) est employé lato sensu pour nommer ce qui relève du groupe linguistique auquel appartient le turc de Turquie (les « langues turkes») et par extension ce qui concerne leurs usagers à l'échelle eurasiatique (les « Turks ») ${ }^{19}$.

\section{La distinction entre turc et turk, conséquence d'un hold up onomastique}

Cette intranquillité sémantique n'a évidemment pas toujours existé, elle s'est même laborieusement imposée. S'il est établi que les Européens ont donné le nom de Turquie (Turchia, Toupkía) à l'Anatolie dès le XII ${ }^{e}$ siècle (Cahen 1988: 103; Deny 1945), il reste que, jusqu'à la création de l'État turc contemporain les orthographes turc et turk étaient indifféremment utilisées, en Europe, pour désigner des langues et des gens de l'Empire ottoman aussi bien que d'«Asie intérieure " (Caucase, Asie centrale, Sibérie). C'est l'adoption du toponyme «Turquie » (Türkiye), d'une part, comme appellation officielle de l'État institué en Anatolie et sur un morceau de la Thrace, au début des années 1920, et conséquemment la désignation de ses ressortissants comme "Turcs» (Türkler), d'autre part, qui ont créé les conditions d'une ambiguïté fondatrice. On peut parler d'un véritable coup de mot, pour reprendre une formule de Henri Meschonnic (Gauthier 2010: 333) - ou même d'une sorte de "word-jacking" (comme on parle, en bon français, de "car-jacking») - de la part des nationalistes turcophones de l'Empire ottoman finissant (les autoproclamés «turquistes»), c'est-à-dire d'une captation stratégique du nom turc au profit putatif d'une fraction [les musulmans (post-)ottomans d'Anatolie et de Roumélie] de l'ensemble des individus susceptibles de s'en revendiquer [les turcophones du continent eurasiatique]. En termes techniques, c'était utiliser le même mot comme hyponyme et comme hypéronyme.

C'est donc à un travail de « désambiguation » taxinomique que se sont progressivement attelés les observateurs du fait turc dans le moment même de la création de la Turquie nationaliste. En toute logique, deux options s'offraient à eux : soit on modifiait le nom de l'hyponyme (on forgeait un nouveau mot pour désigner tout ce qui ressortit à la "Turquie nouvelle») soit on changeait celui de l'hyperonyme (on concevait un autre terme pour la catégorie générique). C'est le second scénario qui s'est imposé : on a gardé « turc » pour ce qui est de Turquie, on a forgé " turcique » et systématisé « turk » pour ce qui tient de l'Eurasie. C'est pourtant la première option qui avait d'abord été tentée. Jean Deny soumettait ainsi en 1926 aux lecteurs du Journal Asiatique le néologisme turquien (calqué sur le glossonyme " francilien ») pour distinguer l'idiome pratiqué en Anatolie des autres variétés (Deny 1926 : 132). De mars 1923 à septembre 1926, le professeur de turc à l'École des langues orientales fut aussi le rédacteur du Bulletin périodique de la presse turque du Quai d'Orsay. Cela lui donna l'occasion de tester sa proposition dans une arène profane, comme en témoigne cet extrait consacré aux échos du congrès de Bakou dans la presse stambouliote (été 1926) : 
La question du remplacement des caractères arabes par les caractères latins a déjà été soulevée à plusieurs reprises mais sans succès [...].

Les décisions du congrès de turkologie, tenu à la fin de février, a [sic] donné du regain d'actualité à cette réforme. Ce congrès, auquel ont pris part les représentants de républiques soviétiques turques, des savants russes, hongrois, allemand et "turquiens" a décidé à une forte majorité de 101 voix contre 7 abstentions (dont celle du délégué d'Angora) d'adopter les caractères latins déjà en usage dans l'Azerbeïdjan et certaines provinces autonomes du Caucase (Tchetcheniskan [sic], Karatchaï) (Ministère des Affaires Étrangères 1926 : 11). quand bien même il est revenu à la charge vingt ans plus tard dans le cadre d'une conférence de l'Institut de linguistique de la Sorbonne (Deny $1950: 17)^{20}$. Mais l'idée a bel et bien survécu chez Louis Bazin, son disciple et successeur rue de Lille :

II y aurait beaucoup à dire sur l'emploi du terme [Türk], comme ethnique au cours des âges. Signalons simplement que l'usage actuel tend à limiter le terme Türk à la désignation de la nation moderne organisée par le génie d'Atatürk, et qui constitue la République de Turquie. Les linguistes emploient aussi, avec extension, le terme de "turc" pour désigner le groupe des langues étroitement apparentées à l'idiome des anciens Türük; certains préfèrent, par un artifice graphique, distinguer entre "turc", langue de la Turquie, et "türk", au sens général. Une certaine confusion règne dans ces emplois, mais le contexte permet toujours au lecteur de s'y retrouver (Bazin 1953 : 322).

Passons sur l'hommage au "génie d'Atatürk », assez typique de la façon dont les turcologues français des années 1930-1980 ont endossé le créationnisme kémaliste (Louis Bazin pointe la confusion mais ne prétend pas la résoudre). L'important est qu'à quelques tâtonnements typographiques près la préoccupation est bien là. Pourtant, aujourd'hui encore, un tel souci de désambiguation est loin d'être universellement partagé, en particulier chez les principaux intéressés.

\section{Türkik-Türkî-Türk : les tribulations du nom turc en turc de Turquie}

En Turquie, les auteurs académiques recourent quelquefois, pour l'acception élargie, aux mots Türkik et Türkî. Cependant l'usage de ces termes reste rare, du fait, notamment, que leur signification n'est pas stabilisée. Si bien que, la plupart du temps, c'est le vocable Türk qui assume les deux significations: cette ambiguïté est problématique et appelle, là encore, un examen philologique. Commençons par Türkik. À notre connaissance, le mot ne figure dans aucun dictionnaire. Sans doute est-ce parce qu'il s'agit d'un emprunt à l'anglais - ce que les puristes ne manquent pas de lui reprocher. Le hasard de recherches sur Internet nous a conduit au forum de discussion Türkçesi Varken («Dites-le en turc! »). Sous le titre «Turkic (Türkik) diller demesek, ne desek?» («Si on ne peut pas dire "langues turciques", alors que dire ?), un certain Yiğit Tulga, qui se présente comme un «ouvrier de la langue ", y proposait ainsi il y a quelques années une série de termes susceptibles de se substituer à l'anglicisme honni (sa prose est elle-même composée dans une langue soigneusement « épurée » de toute composante allogène) (Tulga 2007).

European Journal of Turkish Studies, 24 | 2017 


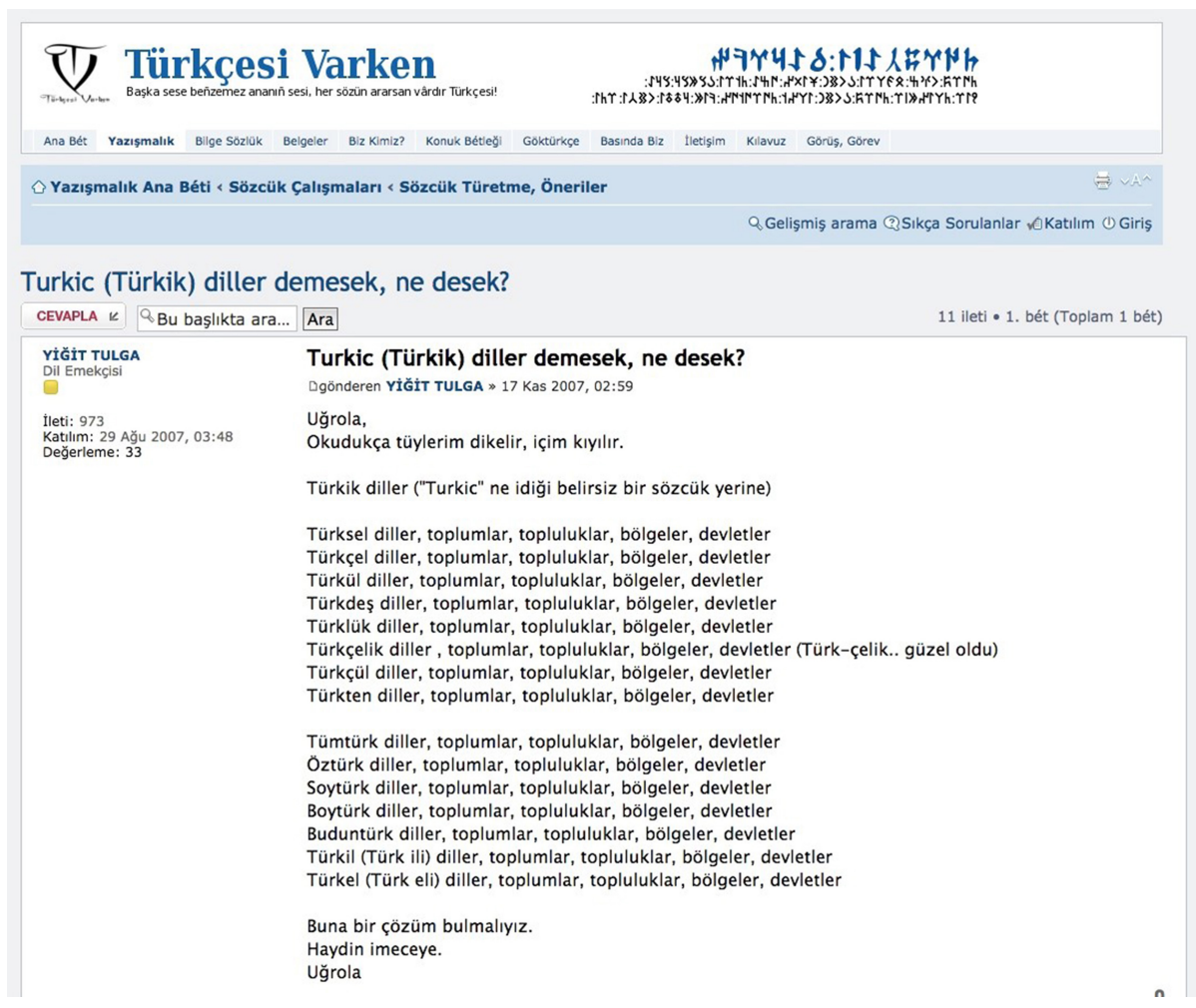

Fig. 2. Post publié sur le forum Türkçesi Varken, le 29 août 2007.

Le post se prête à une analyse structurale. Sur l'axe syntagmatique, on trouve au début de chaque ligne un adjectif générique substitutif à Türkik suivi de la série diller, toplumlar, topluluklar, bölgeler, devletler [x + «langues, sociétés, communautés, régions, États "]. L'auteur, autrement dit, n'est pas seulement en quête d'un glossonyme mais d'un véritable nom propre collectif, y compris ethnonymique. Sur l'axe paradigmatique, défilent une série de néologismes dont le point commun est la répétition de la chaîne de caractères $t-\ddot{u}-r-k$, tantôt prolongée d'un affixe (-sel, -çel, -ül, deş), tantôt précédée d'un substantif convoyant l'idée de totalité (tüm-), de pureté (öz-), de tribalité (boy-) ou d'ethnicité (soy-, budun-) : on retrouve ici le stock lexical et affixal du purisme linguistique turc des années 1930 (l'öztürkçe). En somme, il y a dans cette tentative isolée pour délester le mot Türk de sa trop lourde charge sémantique quelque chose de profondément poétique au sens jakobsonien du terme aussi bien qu'étymologique, en même temps que d'inexorablement nationaliste (Jakobson 2003 : $220 s q$.$) .$

Quant à Türkî, il peut également servir, en turc de Turquie d'aujourd'hui, comme équivalent de notre «turcique " (Türkî diller: «les langues turciques», Türkî cumhuriyetler, « les républiques turciques »); il peut aussi désigner plus spécifiquement la déclinaison orientale du groupe linguistique (le tchagatay). Mais il faut observer, pour commencer, qu'au XIX siècle Türkî, entendu comme raccourci pour lisân-ı Türkî, est le terme par lequel on désigne « la langue turque » souvent de façon indifférenciée, c'est-à-dire telle qu'elle est pratiquée dans l'Empire ottoman aussi bien qu'ailleurs dans le monde turcophone. Ainsi Redhouse traduit indifféremment Türk et Türkî par Turkish (Redhouse 1978). De même, la plupart des usuels publiés à Istanbul dans la seconde moitié du XIX ${ }^{e}$ siècle utilisent le glossonyme Türkî (plutôt que Türkçe), de la Science de la grammaire du turc de Süleyman Pacha (İlm-i Sarf-ı Türk̂̂, 1874) au célèbre Dictionnaire de 
turc de şemsettin Sami (Kamus-ı Türkî, 1900) en passant par les Règles de la langue turque de Halit Ziya [Uşaklıgil] (Kavaid-i Lisan-ı türkî, 1884) (Süleyman Hüsnü Paşa 2006; [Uşaklıgil] 1999 ; şemsettin Sami 2010). Or, il ne paraît pas que cette ambiguïté ait disparu de nos jours. Le Dictionnaire du turc contemporain du TDK donne en effet la définition suivante du vocable Türki : «1. adjectif. Relatif à ce qui est turc [Türkle ilgili]. 2. Nom. Turc [Türkçe].». En résumé, si Türkik est récusé par les puristes en tant qu'emprunt, Türkî a une histoire déjà trop chargée et trop polysémique pour assumer, du moins dans l'immédiat, la valeur du français « turcique ».

De sorte que dans l'immense majorité des cas c'est le même mot Türk que les savants (et les profanes) de Turquie utilisent tantôt au sens étroit, c'est-à-dire civico-politique, tantôt dans l'acception large, ethnolinguistique. La définition de Türk que proposent les sages de la TDK dans le dictionnaire précité illustre parfaitement cette conflation sémantique :

1. nom propre. Peuple [halk] vivant dans les frontières de la République de Turquie et quiconque appartient à ce peuple. « Heureux celui qui se dit turc! (Atatürk) ;

2. ethnie [soy] vivant dans différentes régions du monde et parlant différents dialectes du turc, et quiconque appartient à cette ethnie (« Moi je suis un Turk, ma religion, ma race [cins] sont glorieuses. » M. E. Yurdakul).

Cette ambiguïté a des effets concrets. L'organisation de coopération internationale qui réunit depuis 2009 l'Azerbaïdjan, le Kazakhstan, le Kirghizistan et la Turquie se nomme par exemple Conseil turcique en français et Turkic Council en anglais mais Türk Keneşi en turc (Türk şurası en azerbaïdjanais). Un autre exemple est le célèbre manuel des professeurs Tekin et Ölmez. Curieusement, la première édition portait un titre bilingue français-turc : Türk Dilleri=Les langues turques. À tout le moins est-il ici fait usage d'une forme plurielle (Tekin et Ölmez 1995). Le professeur Ahmet Bican Ercilasun est pour sa part l'auteur d'une synthèse parue en 2004 et plusieurs fois republiée depuis sous le titre Histoire de la langue turque/turke du commencement au XXe siècle. Si l'on en croit le titre du chapitre 2, consacré à "la relation entre le sumérien et le turc/turk» (Sümerce-Türkçe İlişkisi), il faut chercher le terminus a quo de cette «histoire» vers le début $\mathrm{du} \mathrm{II}^{\mathrm{e}}$ millénaire avant notre ère ${ }^{21}$. Pour ce qui est du cadre géographique de l'enquête, il est campé dès les premiers mots du livre :

Il n'est pas facile d'écrire l'histoire d'une langue dont l'existence s'étend sur une surface vaste au point de s'étirer de l'intérieur de la Mongolie et de la Chine jusqu'à l'Europe, de la Sibérie à l'Inde et à l'Afrique du Nord. La langue turque [Türk Dili] qui a laissé des œuvres, des monuments, des inscriptions et des documents par milliers, continue d'être utilisée aujourd'hui au Turkestan, en Azerbaïdjan, en Anatolie, dans les Balkans et dans les steppes de l'Eurasie (Ercilasun $2004: 10$ ).

En résumé, on dira qu'en turc de Turquie le glossonyme Türkçe et l'ethnonyme Türk sont à eux-mêmes leur propre hypéronyme. Une telle oscillation n'est pas anodine. S'il est toujours possible de "s'y retrouver ", comme dit Bazin, l'usage du même mot pour deux réalités distinctes ou plus exactement deux systèmes de mise en ordre de la réalité ne peut manquer d'interroger : une "famille» linguistique transcontinentale et transhistorique d'un côté, une langue récemment nationalisée de l'autre ; des groupes ethniques dispersés à travers l'Eurasie d'une part, une formation nationale d'autre part. C'est un peu comme si l'on désignait par le même vocable le français, le francilien, l'ancien français, le latin, l'italique et l'indo-européen; mais aussi les Français, les Francs et leurs ancêtres les Gaulois et les Celtes. 
Au-delà de ces considérations sémantico-historiques, le problème se pose en termes pratiques : comment devons-nous traduire Türk en français (et dans les quelques autres langues qui admettent une nuance de sens entre " turc » et " turcique ») ? Le problème est particulièrement délicat lorsqu'il se pose dans un contexte énonciatif fortement idéologisé où l'on peut légitimement s'interroger sur la stratégie lexicale du locuteur, c'est-à-dire sur la conscience qui est la sienne de jouer sur et avec les ambiguïtés du mot (un slogan du parti d'extrême-droite nationaliste MHP, par exemple, confondra volontiers les registres civique et racial). Mais on pourrait aussi bien arguer que, d'un point de vue praxéologique, l'écrasement perpétuellement réitéré de deux ordres de réalité aussi hétérogènes dans un seul et même signifiant est toujours problématique, étant entendu que la pratique répétée et banalisée du même mot pour désigner des choses aussi différentes ne peut pas ne pas avoir des effets conatifs profonds sur les locuteurs (Siblot 1999). La question qui se pose est dès lors la suivante: comment rendre en français le chevauchement de la dénotation par la connotation dont le mot Türk est, en turc de Turquie, le théâtre permanent? Ainsi, contrairement à la norme répandue chez les spécialistes (mais répandue depuis peu et seulement chez une partie d'entre eux), nous prenons le parti d'osciller plutôt que de trancher, c'est-à-dire de préférer faire parfois entendre la connotation inévitablement ethniciste et romantique du mot en traduisant Türk par "turc " là-même où une restitution "littérale » ou «turcologique » - mais pas forcément plus scientifique - exigerait qu'on le rendît par «turk».

Traquer « le trafic du sens sous le troc des mots » (Siblot $1999: 29$ ), c'est-à-dire pister les définitions, les usages, les resémantisations pratiques et les avatars plus ou moins synonymiques du signifiant "turcologie ", si ce n'est du nom collectif qui lui sert de racine et de justification, nous a conduit à mettre en lumière un certain nombre de fausses évidences et de vraies confusions sur lesquelles la «science de la turcité » nous semble faire fond. Dans le développement qui va suivre, nous poursuivons l'arpentage $\mathrm{du}$ « domaine " par une réflexion historiographique. Pour comprendre ce que les gens entendent par turcologie, il est en effet pertinent de se demander comment ils en écrivent l'histoire et où ils en situent les gloires.

\section{Comment on écrit l'histoire des études turques}

Voyage en Turcologie : seconde partie. Que l'on soit ou non réfractaire à l'idée même d'une poursuite des commencements en histoire (Chartier 1998), il est un fait qu'un examen de la littérature rend rapidement évident : la quête de l'origine des études turques se prête à des opérations de savoir à la fois nombreuses et diversifiées. Il vaut la peine de les thématiser dans un dossier de revue qui, lui-même, se propose de penser les dynamiques à l'œuvre dans l'histoire du "domaine " à partir d'une pluralité de lieux, d'expériences et d'acteurs. La question est donc la suivante : que disons-nous de la turcologie - et que faisons-nous en le disant - lorsque nous entreprenons d'en raconter l'histoire? Sans prétendre à une exhaustivité de toutes façons illusoire, nous examinerons dans le développement qui va suivre différentes manières de faire de l'histoire des études turques, qui inscrivent ces dernières dans une multiplicité de passés et d'historicités possibles. Trois tropismes se dégagent particulièrement que nous identifions sous le triptyque suivant : nativisme, eurocentrisme, millénarisme. 


\section{Nativisme. La turcologie est (presque) aussi ancienne que les Turks}

La perspective nativiste consiste à faire commencer les études turques, si ce n'est avec "les Turcs eux-mêmes» (toujours entendus comme une entité stable à travers l'histoire), du moins avec telle de « leurs » œuvres particulièrement remarquable. Ainsi l'entrée «Turkology » de Wikipédia (édition anglophone) fait remonter les origines du domaine au très célèbre Dictionnaire de la langue turque de Mahmoud de Kachgar : «Des données ethnographiques sur les tribus turkes furent assemblées de façon systématique pour la première fois par le philologue turk Mahmoud de Kachgar dans le Dīwān ul-Lughat it-Turk [...] » ${ }^{22}$. C'est au même Kachgari que le professeur Kocaoğlu de l'université Koç d'Istanbul attribue le titre de " premier turcologue connu » (Kocaoğlu $2004)^{23}$. Même son de cloche chez son collègue Bekir Yavuz Pekaçar, chargé de cours à l'université Gazi d'Ankara (2012) : «À la lumière des documents disponibles, le premier turcologue d'extraction turque que nous connaissions (bildiğimiz ilk Türk kökenli Türkolog) est Mahmoud de Kachgar, qui a vécu à l'époque des karakhanides [...]» (Pekaçar 2012 : 257).

Prenons l'argument au sérieux. Composé à Bagdad dans les années 1072-1074 par un noble ouïghour lié à la dynastie karakhanide et dédicacé au calife Al-Muqtadi, le Dīwān de Kachgari prodigue en arabe, à l'intention des élites abbassides, et conformément à la tradition lexicographique arabe, un lexique de plus de 8000 vocables accompagné d'un vaste compendium des savoirs du temps sur les peuples turkophones ${ }^{24}$. L'ouvrage comprend en outre un exposé de la syntaxe du turk qui nous est connu indirectement (Ermers 1999: 17), ainsi qu'une carte présentée ci-dessous. En substance, le Dīwān est d'abord et avant tout, à l'intention des élites arabes et dans leur langue, une œuvre de défense et illustration de la gloire des Turks, que l'auteur assure avoir visités de la Chine septentrionale à la Mésopotamie. Si les karakhanides et leur dialecte princier apparaissent sous sa plume comme les plus raffinés des Turks, ce sont les tribus oghouz qui retiennent particulièrement l'attention de l'auteur: ces "gens du voyage" récemment débarqués de l'intérieur du continent et fraîchement convertis à l'islam qui ont fini par fonder au cœur du vieux pays arabo-persan le nouvel empire seldjoukide avant de ravir l'Asie mineure aux Byzantins ${ }^{25}$. 


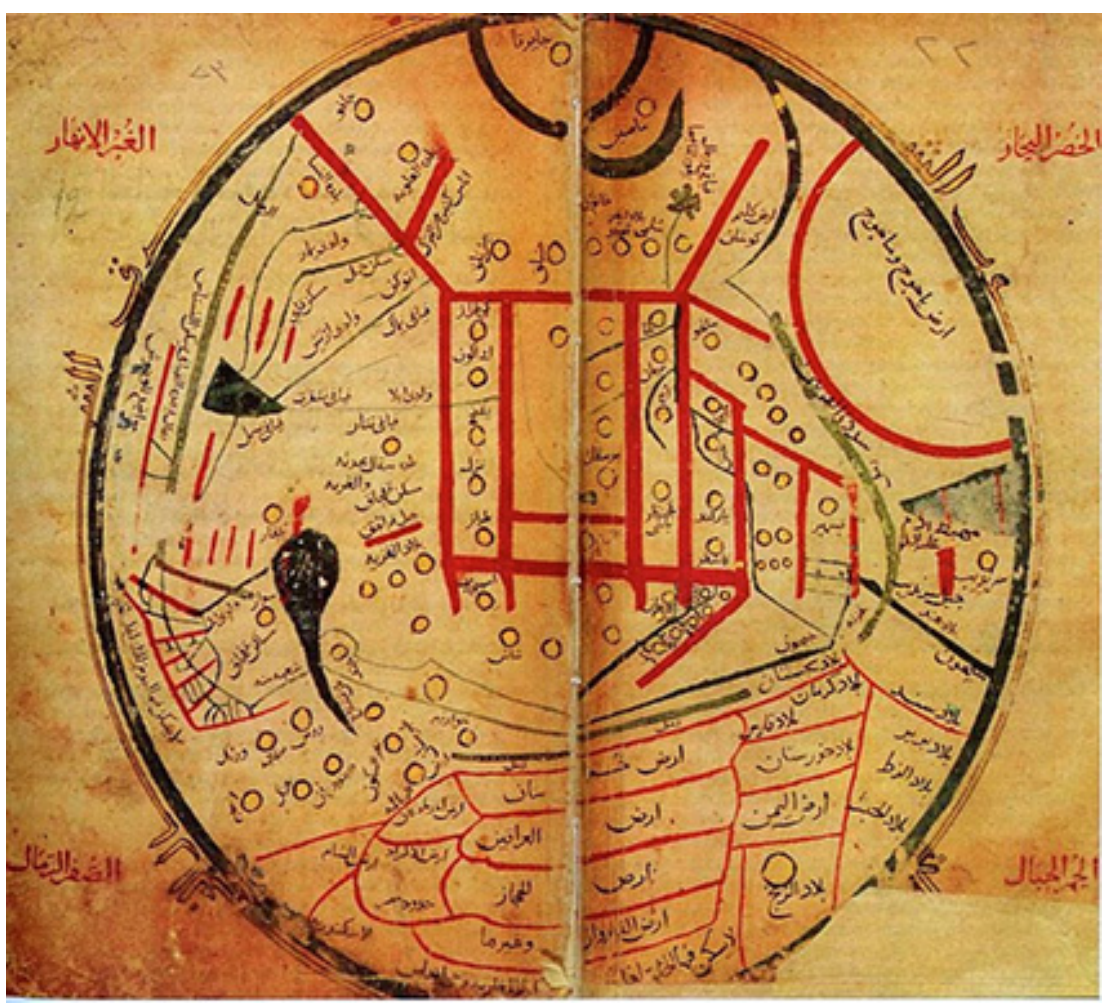

Fig. 3. Le monde turk selon Mahmoud de Kachgar.

Bibliothèque nationale, Manuscrits (Istanbul) ${ }^{26}$.

$\mathrm{Nul}$ doute que pour Kachgari le monde turk constitue un tout : son projet est de décrire ce dernier comme un ensemble linguistique et culturel cohérent, par-delà les frontières confessionnelles de l'Oumma (Kaplony 2008 : 144). « Kāshgarī est parvenu à faire pour les Turcs, écrit ainsi Robert Dankoff, ce que les philologues arabes des premiers siècles de l'Islam avaient accompli pour les Arabes, à savoir : produire une présentation claire et distincte de leurs traditions linguistique, généalogique et culturelle " (Dankoff 2008a : 15). En témoigne également la carte que l'auteur joint à la section géographique de l'introduction du livre. Centrée sur la ville karakhanide de Balasagun (actuel Kirghizistan), celle-ci frappe d'emblée par son amplitude. L'unique échantillon que nous possédions (qui fut recopié et « actualisé » à Damas dans les années 1260) court de l'Afrique du nord au Japon, de l'Inde à la mer d'Aral. Quant aux points jaunes qui clairsèment la feuille, ils représenteraient les différents groupes turks identifiés au fil du livre, depuis la "Grande Chine », vers le Levant, jusqu'au " pays de Roum », au Couchant (Kaplony 2008 : 147 sqq.). L'œuvre, résumait René Giraud, « est plus qu'un dictionnaire. Elle est tour à tour une grammaire comparée des dialectes turcs du temps, un traité de sociologie, une anthologie de poèmes et de proverbes, une géographie, bref une œuvre vivante à laquelle la turcologie doit une grande part de ses progrès récents »(Giraud 1960 : 21). Et pour cause : si Kachgari est connu des Ottomans et pratiqué jusqu'au XVII ${ }^{\mathrm{e}}$ siècle, notamment par l'encyclopédiste Katip Çelebi, son œuvre sombre par la suite dans l'oubli.

Ce n'est qu'à la veille de la Grande Guerre qu'un manuscrit de plus de 600 pages, abîmé et désordonné mais complet (dont est tirée la carte supra), est découvert au hasard d'une flânerie sur le marché aux livres de Beyazit. Son inventeur est Ali Emîrî, 
fonctionnaire des impôts en retraite, collectionneur bibliophile et fin lettré. Il confie la préparation de l'édition du manuscrit à son ami Kilisli Rifat, professeur d'arabe, de persan et de théologie en médressé. La nouvelle ne tarde pas à s'ébruiter dans la capitale ottomane, notamment au sein d'une toute nouvelle société savante dont Ali Emîrî est proche : la Société pour l'étude des œuvres islamiques et nationales (Asar-l islamiye ve milliye tetkik encümeni) ${ }^{27}$. Créée en 1915 , cette organisation officielle est importante pour notre propos : si les clubs nationalistes de l'époque unioniste avaient en effet déjà adopté un agenda turquiste-turcologique, aux confins de la science et du militantisme nationaliste, celle-là peut être considérée comme la première académie ottomane d'études turques, bien que sans le nom, rappelle François Georgeon, le grand vizir Said Halim Pacha ayant refusé l'intitulé «Société de turcologie » que réclamaient pour elle les professeurs Köprülüzâde et Ziya Gökalp ${ }^{28}$. Comme le rappellent encore Aykut Kazancigil et Ayhan Vergili, la Revue des études nationales (Millî Tetebbular Mecmuasl) publiée par la société présente d'ailleurs toutes les caractéristiques d'une revue internationale de turcologie, et cela une décennie avant la création, par le même Köprülüzâde, de la célèbre Türkiyât Mecmuası, à laquelle on attribue parfois le titre de première revue d'études turques (Kazancigil et Vergili 2013). Ainsi le sémitisant allemand et professeur au Séminaire des langues orientales de Berlin Martin Hartmann y publie dès l'automne 1915 un article sur Mahmoud de Kachgar ${ }^{29}$.

Finalement édité en trois volumes à Istanbul (1917-1919) (Kilisli Muallim Rifat 1333-1335), l'opus magnum de Kachgari fait aussitôt son entrée dans la bibliothèque orientaliste internationale. Le comparatiste Carl Brockelmann, professeur à l'université de Halle puis de Berlin, lui consacre deux articles en 1919-1920, avant de faire paraître un index des termes turks de l'ouvrage qui d'emblée devient un usuel (Brockelmann $1928)^{30}$. La publication du livre vient surtout dynamiser une turcologie turcophone dont l'institutionnalisation est étroitement liée aux stratégies de légitimation du nouvel État turc, tant vis-à-vis de sa propre population qu'à l'international. L'ouvrage nourrit ainsi les travaux de Köprülüzâde, Necib Asım, Avram Galanté, Zeki Velidi Togan ou encore Ahmet Caferoğlu (qui est l'étudiant de Brockelmann à Berlin de 1925 à 1929) ${ }^{31}$. Au même moment, Jean Deny fabrique à Paris son propre fichier lexicographique (fig. 4) (32 $^{32}$ La première traduction du Diwan en turc, accompagnée d'un nouvel index, est publiée en 1940-1941 par le trésorier du TDK et député à la grande assemblée nationale d'Ankara Besim Atalay ${ }^{33}$. 


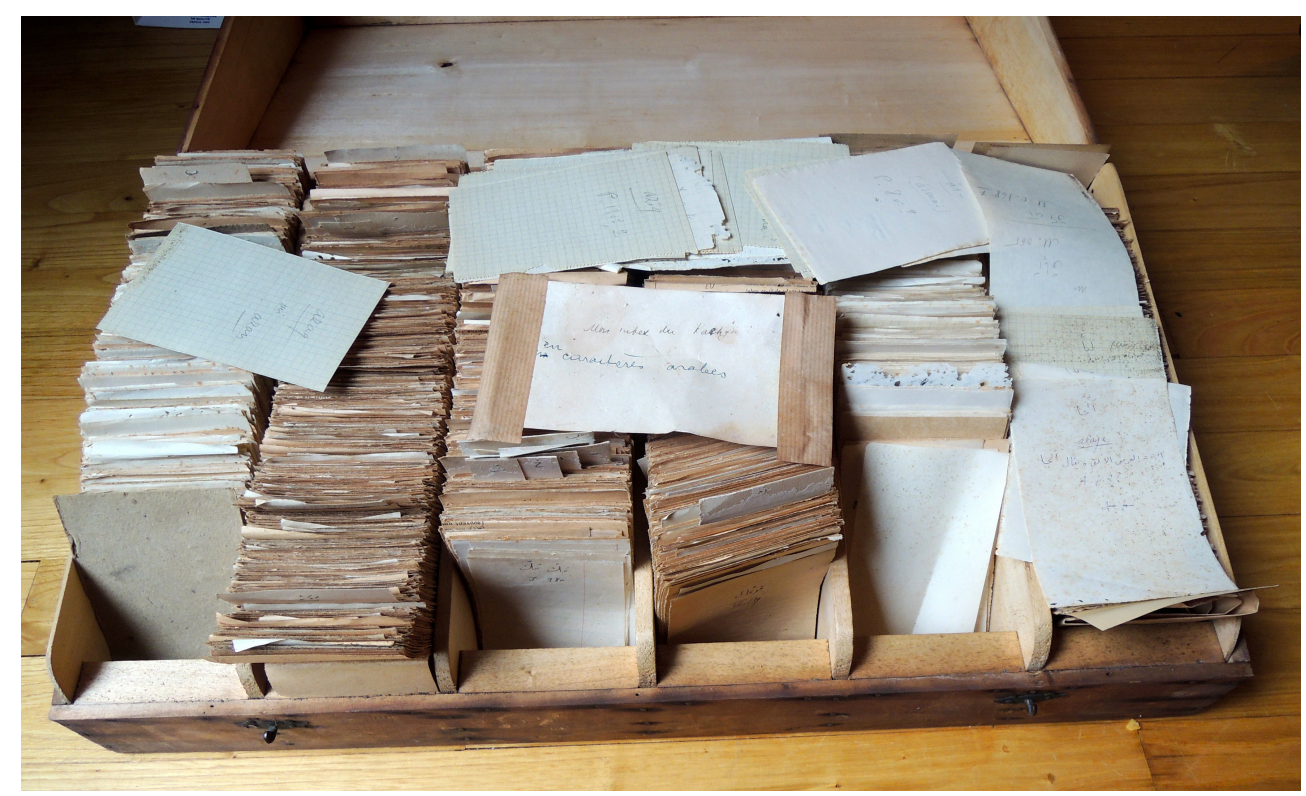

Fig. 4. « Mon index du Kachgarî. En caractères arabes ».

Fonds Jean Deny (BULAC, Paris).

En somme, il n'est pas exagéré d'affirmer que «l'invention de Kachgari» dans la première moitié $\mathrm{du} \mathrm{XX}^{\mathrm{e}}$ siècle $\mathrm{a}$ autant contribué à dynamiser l'intelligence turcologique internationale - mettre le monde turk en mots - qu'à stimuler des pratiques et des références emblématiques de l'orientalisme philologique (la constitution d'index et de fichiers). Comme le montre la fig.4, l'architexte que constitue le manuscrit est à l'origine de la création et de la conservation d'une multitude d'archives à travers le monde turcologique. Enfin, redisons que la découverte du manuscrit de Mahmoud de Kachgar en pleine guerre mondiale arrivait à point nommé. Vingt ans après l'émotion suscitée par le déchiffrement des stèles de l'Orkhon (qui renvoyaient, elles, à un passé pré-islamique), et à l'heure où la Turquie cherchait ses marques territoriales et symboliques dans un nationalisme à forte connotation religieuse, ses apologues ne pouvaient qu'accueillir avec enthousiasme un texte qui venait si opportunément accorder au nom turk un nouveau gisement de respectabilité historique. Les raisons sont nombreuses, en définitive, pour voir en Kachgari une sorte d'«architurcologue ». On ne voit guère au nom de quel présentisme il faudrait toutes les récuser.

\section{Eurocentrisme. La lumière vient d'Occident}

37 Les orientalistes, en particulier (mais pas uniquement) les orientalistes européens, adoptent souvent un point de vue eurocentrique sur la généalogie des études turques. Celui-ci consiste à faire coïncider les débuts de la turcologie, soit de manière explicite, soit par simple effet de cadrage analytique ou narratif, avec l'arrivée des Turcs sous le regard des Européens. D'aucuns seraient tentés de dénoncer cet ethnocentrisme comme l'expression d'une colonialité mal digérée (Ahıska 2008 : 146). Il est plus intéressant, à notre sens, de rappeler ce que de telles narrations historiographiques présupposent quant à la circonscription même de l'objet turcologie. Reste à s'entendre sur les mots : par Turcs la plupart pensent Ottomans et font dès lors commencer les études turques au 
moment où l'Empire éponyme apparaît dans les sources européennes (Bossaert 2016). Eurocentrisme rime alors avec ottomano-centrisme.

L'article précité de Babinger sur « l'histoire des études turques en Europe » s'ouvre par l'évocation du voyageur bavarois Schiltberger à la cour de Bayazid II puis de Tamerlan (XV ${ }^{\mathrm{e}}$ siècle) (Babinger 1919). Dans son essai «Herméneutique, orientalisme, turcologie », Halil İnalcık associe pour sa part « le commencement de la turcologie en Europe " ( Avrupa'da Türkolojinin başlangıçı») aux controverses qui agitent la chrétienté germanique après la chute de Constantinople (İnalcık 2002 : 25). De même, nombreux sont ceux qui situent le terminus a quo de la turcologie à la Renaissance. Convié par Köprülüzâde à donner une conférence à la Maison du Peuple lors d'un passage à Istanbul, le professeur de l'université de Rome Ettore Rossi livre une généalogie des "études de turcologie en Italie » qu'il fait commencer avec le voyageur Pietro della Valle $\left(\mathrm{XVI}^{\mathrm{e}}\right)$ (Rossi $\left.1933: 511\right)^{34}$. Attelé à écrire l'histoire du domaine dans son pays, Louis Bazin pense de même que « la turcologie s'est développée en France à partir du XVI ${ }^{\mathrm{e}}$ siècle, quand l'Empire ottoman était à son apogée » (Bazin 1994: 414). Même son de cloche chez Gilles Veinstein : "On serait tenté de faire remonter la naissance des études turques en France à la fameuse alliance entre François $\mathrm{I}^{\mathrm{er}}$ et Soliman le Magnifique [...]» (Veinstein 2014 : 309). C'est en Guillaume Postel, l'auteur de De la République des Turcs $(1560,1575)$ et son lointain prédécesseur au Collège de France, que ce dernier identifie «les débuts, modestes mais mémorables, de notre turcologie " (Veinstein 2000: 2). Auteur de l'entrée "turcologie» du Dictionnaire de l'Empire ottoman, François Georgeon, admet pour sa part que c'est «en Europe que l'intérêt pour la langue turque s'est d'abord manifesté ». Il cite en exemple le Thesaurus linguarum orientalium (1680) du Lorrain installé en Pologne François Meninski et la Grammar of the Turkish Language (1832) du Britannique Arthur Lumley Davids ; " [en revanche] dans l'Empire, il faut attendre les Tanzimat pour voir apparaître les premiers travaux sur la langue turque", explique encore l'auteur, qui pense tout particulièrement aux célèbres Kavaid-i Osmaniye de Cevdet et Fuad Pacha (1851) (Georgeon, Vatin et Veinstein 2015).

Tout se passe en somme comme si l'Europe était l'institutrice de la turcologie. Et qu'à ce titre elle avait "inventé », au sens scientifique du mot, la langue turke si ce n'est les Turks eux-mêmes. Et pour cause : quand on connaît le poids immense que la somme des savoirs philologiques, ethnographiques, historiques et folkloriques produits sur le monde turk dans l'Europe des XVIII ${ }^{\mathrm{e}}$ et XIX ${ }^{\mathrm{e}}$ siècles a pu avoir sur la cristallisation de l'imaginaire national turc, tel qu'il s'est formé dans l'Empire ottoman à partir des années 1860 , on est tenté de faire du turquisme - et par ricochet de la Turquie ellemême, comme projet national devenu réalité politique - un produit dérivé de la turcologie. "Chose étonnante finalement, écrit par exemple l'historien Halil İnalcık au début de son "Herméneutique ", ceux qui sont arrivés à la conscience de la turcité (Türklük bilinci) ont trouvé les sources de l'idée nationale dans la turcologie française, anglaise et hongroise » (Inalcık $2002: 28$ ).

Cette idée d'antécédence et de dépendance de la Turquie vis-à-vis de la turcologie ne date pas d'hier. On la rencontre déjà sous la plume du sociologue et théoricien nationaliste Ziya Gökalp dont les Principes du turquisme (1923) sont souvent présentés comme la «Bible» du nationalisme turc dans sa variante républicaine. En voici les premiers paragraphes : 
Avant que le turquisme ne fasse son apparition dans notre pays, deux processus ont pris corps en Europe concernant la turcité. Le premier est ce que l'on appelle en français turquerie, c'est-à-dire la turcophilie. Les tissages de soie ou de laine, les tapis, les kilims, les céramiques, les pièces de fer forgé ou d'ébénisterie, les œuvres de nos maroquiniers et de nos enlumineurs, les poêles (mangal), les chandeliers et autres œuvres d'art turc fabriquées en Turquie ont depuis longtemps attiré l'attention des amateurs de belles choses en Europe. [Aux amateurs de turqueries, l'auteur associe toute une série d'écrivains, de Lamartine à Claude Farrère en passant par Auguste Comte et Pierre Loti].

On donne au second mouvement apparu en Europe le nom de turcologie (Türkiyât). En Russie, en Allemagne, en Hongrie, au Danemark, en France, en Angleterre, de nombreux hommes de science ont commencé à mener des recherches historiques et archéologiques (tarihî ve atikiyatî taharriler). Ils ont mis en évidence le fait que les Turcs sont une nation très ancienne, qu'elle s'était étendue sur d'immenses territoires et qu'à différentes époques elle a donné vie à des États d'envergure mondiale (cihangirane devletler), ainsi qu'à de hautes civilisations. En vérité, l'objet des études en question n'était pas les Turcs de Turquie mais les anciens Turcs orientaux. Mais, tout comme le premier mouvement, ce second mouvement n'a pas été sans marquer l'esprit de certains penseurs dans notre pays. Ce sont surtout la grande histoire écrite par l'historien français de Guignes sur les Turcs, les Huns et les Mongols et la grammaire générale du turc publiée par le savant anglais Sir Lumley Davids sous le titre Le Livre de la connaissance utile offert au sultan Sélim III (Sultan Selim-i Sâlis'e ithaf ettiği (Kitab-ı ilmü'n-Nafi) ismindeki umumî Türk sarfï ${ }^{35}$ ) qui ont laissé des traces profondes sur l'esprit de nos penseurs. [...] Dans cet ouvrage, il était question, outre les différentes branches de la langue turque, de la civilisation turque, de l'ethnographie et de l'histoire turques (Gökalp $1972: 2$ ).

41 Si le livre de Gökalp est une pierre fondatrice du turquisme, il est aussi un autel à la gloire de la turcologie européenne. Le texte campe une sorte de panthéon des études turques que l'on retrouve fréquemment dans les récapitulations du domaine. Il reste qu'il opère un glissement par rapport aux généalogies précitées : plutôt qu'à l'époque de la Renaissance et de la montée en puissance des Ottomans, c'est à l'époque moderne qu'il fait naître la turcologie, quelque part entre les investigations ethnographiques d'un Joseph de Guignes sur l'Asie centrale (au XVIII ${ }^{e}$ siècle) $)^{36}$, et la (re)formalisation de l'idée d'une continuité ethnolinguistique telle qu'on la trouve dans la Grammaire de la langue turke du Britannique Arthur Lumley Davids $(1832,1836)$ :

Pendant que les 'Osmânlî civilisés font des pas rapides pour rivaliser avec les plus grands savants et les plus polis des états européens, leurs frères errants à l'extrême Nord, et dont la langue est l'unique signe de leur parenté, se trouvent plongés dans l'abîme de l'ignorance et de la barbarie primitives, formant les deux extrémités de cette longue chaîne de société et de civilisation, dont les anneaux sont régulièrement formés par les diverses nations intermédiaires d'origine turke (Davids $1836: 2$ ).

Enfin, certains font plutôt naître la turcologie dans la seconde moitié du XIX ${ }^{\mathrm{e}}$ siècle. La période marque en effet un jalon intellectuel avec la parution des travaux de Wilhelm Radloff à Saint-Petersbourg, d'Arminius Vambéry à Budapest, d'Ahmet Vefik Pacha à Istanbul, de Theodor Zenker à Leipzig et d'Abel Pavet de Courteille à Paris. Ainsi a-t-on pu dans les liens épistolaires entre un Redhouse, un Radloff et un Vambéry comme les effets à l'œuvre d'une " communauté disciplinaire » (Espagne 2013: 81). Biographe de Wilhelm Radloff, Ahmet Temir rapporte la revendication de ce dernier qui, au soir de sa vie, réclamait pour lui d'avoir été le "premier des turcologues» (Temir 1991: 67). On sait en effet à quel point la découverte, puis le déchiffrement, des stèles de l'Orkhon, dans les années 1890, sont venus conforter la conviction, chez bien des hommes de 
lettres qu'un savoir nouveau, si ce n'est une science neuve, avait vu le jour. L'un de ses vulgarisateurs, le Français Léon Cahun, s'en avise d'ailleurs dans sa célèbre Introduction à l'histoire de l'Asie :

S'il n'est pas possible d'écrire l'histoire de l'Asie au moyen âge sans exposer celle des Turcs, il est facile de le faire en la racontant. D'autre part, les notions insuffisantes ou fausses qu'on avait sur le passé de ces peuples, ont été modifiées du tout au tout, pendant ces trente dernières années, par des découvertes remarquables et par des travaux de premier ordre (Cahun $1896: 33$ ).

Dans cette perspective, évoquons encore Yusuf Akçura. François Georgeon a souligné à quel point l'École libre des sciences politiques, où le futur théoricien du (pan)turquisme étudia de 1899 à 1903, fut pour lui, autant que de formation intellectuelle, un lieu de théorisation si ce n'est d'apprentissage identitaires (Georgeon $1980: 22 \mathrm{sq}$.). On peut en dire autant de l'École pratique des hautes études où le jeune Tatar assiste au séminaire du sémitisant Joseph Halévy. Il y est accompagné par son compatriote Sadri Maksudi qui comme lui deviendra un intellectuel organique du régime kémaliste :

\begin{abstract}
À la fin de chaque leçon, écrit ce dernier, Halévy nous prenait à part, Yusuf et moi, et nous faisait l'éloge des anciens Turcs. J'ai encore en mémoire certaines de ses paroles : « Lorsque les inscriptions de l'Orkhon ont été gravées (732-734 ap. J.-C.), aucune des langues actuelles de l'Europe occidentale n'était encore formée; et cependant, déjà à cette époque, vos ancêtres à vous possédaient une langue littéraire capable de tout exprimer. Votre culture est plus ancienne que celle de n'importe quelle nation d'aujourd'hui ; vous pouvez être fiers. » (Maksudi 1977 : 347, cité dans Georgeon $1980: 32$ ).
\end{abstract}

La fabrique turcologique de la fierté d'être né turc est donc un billard à bandes multiples : ici, deux Tatars de Kazan, ayant fait leurs études secondaires qui à Istanbul qui en Crimée, sont sensibilisés, au cours de leur études à Paris, à la découverte d'artéfacts épigraphiques déchiffrés au sud du lac Baïkal une décennie auparavant par un savant danois (Wilhelm Thomsen) et un Russe d'origine allemande (Wilhelm Radloff). En somme, c'est un peu comme si, à l'époque de la Renaissance, au temps des Lumières, dans la première moitié du XIXe siècle, ou quelque part entre la fin des années 1860 et l'orée du XXe siècle, une « révélation turcologique » était venue - mais toujours aux Européens d'abord.

\title{
3. Millénarisme. La turcologie n'est pas encore née
}

Certains philologues situent pourtant les origines des études turques très amont de la traversée des Dardanelles par les Fils d'Osman. Voici par exemple comment Hasan Eren retrace l'histoire du domaine dans son Dictionnaire de la turcologie (Eren 1998), ouvrage dont le premier et unique tome est consacré aux "turcologues étrangers » (yabancı Türkologlar ${ }^{37}$ ) :

Les racines de cette branche scientifique à laquelle on donne le nom de science du fait turc [Türklük bilimi] remontent à l'Europe du XIVe siècle. Toutefois, si l'on admet une acception large de cette expression entendue comme «branche scientifique faisant mention des Turcs » [Türklerden söz eden bilim kolu], il est possible de considérer les écrivains latins et byzantins ayant produit des informations sur les anciennes tribus turques [eski Türk boylari] comme des précurseurs de la science du fait turc. Toutes les nations européennes ont recueilli des connaissances des anciens Turcs [eski Türkler] d'écrivains comme Ammien Marcellin (IV ${ }^{e}$ siècle), Priscus (mort vers 472 ), Sidoine Apollinaire (IV siècle), Jordanès ( $\mathrm{VI}^{\mathrm{e}}$ siècle), 
Procope (VI siècle), Ménandre le Protecteur (VI ${ }^{\mathrm{e}}$ siècle) ou encore Grégoire de Tours (mort en 594) (Eren 1998 : 23).

Une obsession de l'enracinement traverse cet extrait. Tout se passe comme si, pour donner leurs lettres de noblesse aux études turques, il fallait leur faire rejoindre l'Antiquité. Mais l'on remarquera que, dans le même temps, cette anamnèse a pour corollaire un élargissement de la surface couverte par la turcologie et une redéfinition de son objet même: elle n'est plus tant un champ de savoirs travaillés par des disciplines qu'une compilation de mentions documentaires. Et pour être européenne, la «science du fait turc » n'est plus la lumière venue d'Occident qui, jusque dans le tréfonds de leur ontologie, révèle les Turcs à eux-mêmes, que le réceptacle passif de leurs pérégrinations historiques.

Quitte à s'affranchir de la chronologie, il est possible d'aller encore un peu plus loin. Et d'arguer que, tout bien pesé, la véritable turcologie n'est pas encore née. Les fameuses «thèses » d'histoire et de linguistique, qui tiennent lieu de vérité officielle dans la Turquie des années 1930, ne sont pas loin de considérer les choses ainsi. Résumons grossièrement : en se dispersant à la surface du monde depuis l'Asie centrale, les Turcs préhistoriques ont fait don du langage, et de la civilisation même, à l'ensemble de l'humanité. Toutes les grandes civilisations de l'histoire, des Chinois aux Mayas en passant par l'Égypte pharaonique et la Grèce antique, trouvent leur source dans le génie de la race turque préhistorique, dite « race alpine » (Beşikçi 1991; Ersanl1 1992 ; Copeaux 1997 ; Copeaux 2000). De même, le turc est la source de toutes les langues « civilisées » (c'est-à-dire sémitiques et indo-européennes), si ce n'est la langue-mère de l'humanité (Aytürk 2004 ; Szurek 2017). Une turcologie conséquente ne peut dès lors que tutoyer l'universel. Et s'annexer, chemin faisant, la prétention nomothétique d'autres disciplines des sciences humaines et sociales, en particulier la linguistique et l'anthropologie.

C'est ainsi qu'un manuel destiné aux étudiants de la faculté de lettres, histoire et géographie d'Ankara traitant de la naissance du langage articulé chez l'homme préhistorique paraît en 1936 sous le titre Notes sur la théorie de la langue-soleil. Turcologie II (İnan 1936b). «Turcologie », ici, n'est pas tant le nom d'un domaine idiographique (l'étude d'une singularité linguistique, historique, ethnique ou culturelle) que celui d'une science humaine. Mieux : de la science de l'Homme. Précisons que l'auteur du manuel, Abdülkadir İnan, n'est pas le premier venu mais un turcologue patenté. Tatar de Bachkirie (comme Zeki Velidi Togan auquel il est lié), fils d'imam (comme Sadri Maksudi), il a commencé sa carrière d'enseignant dans les établissements secondaires musulmans de l'Empire tsariste. Après la Première Guerre mondiale, il prend part aux luttes indépendantistes des musulmans de Russie. La victoire des bolcheviks le contraint à l'exil : il gagne l'Inde en 1924, Marseille, Paris puis Berlin en 1925 (Tülücü 2000). Comme beaucoup de musulmans de l'Empire russe ayant vu s'accomplir sur le terrain anatolien leur espoir de création d'une république turcique indépendante (Eissenstat 2002 : 47), il arrive en Turquie en 1925 où il devient l'assistant de Mehmet Fuat Köprülüzâde, directeur du nouvel Institut de turcologie d'Istanbul. Son nom figure dans la première livraison de la Türkiyat Mecmuası où il publie un long article sur le Livre de Dede Korkut ainsi qu'une note sur « les noms de tribus turques ${ }^{38}$. Sa contribution personnelle à la turcologie turque, notamment en tant que traducteur des linguistes et turcologues soviétiques, lui vaut un poste à la nouvelle université d'Ankara. 
Pour comprendre ce que la théorie de la langue-soleil est censée représenter dans l'histoire des études turques, reportons-nous au Précis de turcologie qu'Abdülkadir İnan fait paraître la même année (İnan 1936a). Ce manuel s'ouvre par les propos suivants :

Notre sujet est la langue turque. La langue turque, ce n'est pas seulement la langue parlée en Turquie. C'est une langue répandue sur une surface immense qui s'étend des rivages de la mer d'Okhotsk [dans l'océan Pacifique], en Orient, jusqu'à la Vistule en Pologne, à la Méditerranée, au bassin du Danube, jusqu'aux monts de l'Hindou-Kouch et au golfe de Bassorah au sud, et à la mer de glace [l'océan Arctique] vers le nord. Il est même attesté que, dans l'ancien temps [eski devirde ise], la langue turque, en tant que langue de culture, couvrait l'ensemble de l'Asie, de l'Afrique et de l'Amérique. Il reviendra à nos amis d'étudier et d'expliquer ce problème. Dans le cadre de ce cours, notre sujet sera le turc d'aujourd'hui (İnan 1936a:3).

Pourtant l'auteur y revient. Quelques pages plus loin il propose en effet un " historique » (tarihçe) de la turcologie fondé sur une périodisation en trois temps, qu'il déclare emprunter à son confrère russe Samoïlovitch. La première période est celle de la "turcologie d'Asie ancienne » (eski Asya Türkoloji) : elle remonte à quinze siècles en arrière, c'est-à-dire aux chroniques de la Chine ancienne, lesquelles contiennent « de nombreuses connaissances sur le mode de vie des Turcs, leurs croyances, leurs cérémonies mortuaires, leurs rituels religieux et leurs accoutrements " (Inan 1936a : 13); elle intègre aussi le temps de "l'invasion arabe» et "mongole», et la vaste production philologique correspondante. Le livre de Kachgari, "véritable trésor pour les turcologues ", illustre l'acmé de cette très longue première période. La suivante est celle de la turcologie " centre-européenne » (Orta Avrupa devri). Elle commence avec le Codex Comanicus (XIV ${ }^{\mathrm{e}}$ siècle), se développe à la faveur de la crainte qu'inspirent aux Européens les Ottomans, pour atteindre sa " perfection » avec Radloff et Thomsen (fin $\mathrm{XIX}^{\mathrm{e}}$ ). Le troisième et dernier âge (ou "New Age», yeni devir), c'est l'époque contemporaine, qui voit les Turcs se placer «à égalité » vis-à-vis des savants européens :

L'événement le plus important de cette période c'est la théorie de la langue-soleil que le génie turc a mise au jour, laquelle sera considérée comme un tournant non seulement pour la turcologie mais aussi pour la linguistique générale. [Et l'auteur de se répéter :] Il ne fait aucun doute que cette période constituera l'une des plus brillantes dans l'histoire non seulement de la turcologie mais de la linguistique générale et de la philologie (İnan 1936a : 14 sq.).

51 Autrement dit, la turcologie entre dans sa phase nomothétique. Il faut de ce point de vue prendre au sérieux les propositions que Hasan Reşit Tankut, secrétaire-général du TDK, formule en français dans une brochure intitulée «L'histoire par la linguistique. Le mot "Alp" et le foyer de la race alpine » (1938). En voici les premiers paragraphes :

L'horizon, vaste comme le monde, qu'a déployé la thèse historique turque a ouvert la voie à l'université $[s i c]$ de la thèse linguistique turque. Si la science linguistique turque peut projeter sa lumière jusque sur la préhistoire, c'est grâce à la conquête de l'origine de l'homme par la science historique turque. [...]

[À l'inverse,] Si la science historique turque parvient à connaitre le premier homme, c'est parce que la science linguistique turque a pour ainsi dire doté de la parole les fossiles des mots. Car l'œuvre infaillible du Génie turc, la théorie de Soleil-Langue, a découvert et mis au jour le premier fragment de son de l'homme, le premier mot comportant un sens qu'il ait prononcé. [...]

La parole est un miroir dans lequel nous voyons l'aspect d'ensemble de celui qui le prononce. C'est pourquoi les sciences historique et linguistique, bien que distinctes l'une de l'autre comme le sont l'homme et la parole, fonctionnent et vivent toujours 
à l'émisson [sic]. Les branches auxiliaires de l'une et de l'autre sont, elles aussi, soumises à cette collaboration. N'est-ce pas pour cette raison que l'on donne le nom d'« Anthropologie » à l'ensemble des sciences qui se rapportent à l'anthropos?

Les sciences historiques et linguistiques turques, ces deux sœurs jumelles, se trouvent ainsi avoir entrepris d'édifier l'anthropologie turque. Du reste, la valeur et l'intérêt égaux que le grand Libérateur Atatürk, qui est aussi le créateur de cette science, accorde à tout ce qui touche à l'individu Turc ne sont-ils pas une manifestation symbolique de l'anthropologie prise dans un sens fort large? (Tankut $1938: 3)$.

De cette "anthropoturcologie " on trouve déjà trace au XVIII ${ }^{\mathrm{e}}$ congrès international des orientalistes qui se réunit à Leyde en septembre 1931. Il vaut la peine de s'arrêter sur la séance du 10 septembre au matin qui, sous la présidence de Vladimir Minorsky ${ }^{39}$, est en bonne part consacrée au domaine turk. Si cette séance prend place dans une section "Asie antérieure et centrale ${ }^{40}$ ", elle n'en donne pas moins lieu, de la part des personnalité réunies, à une véritable mise en scène de leur appartenance collective à la turcologie internationale. Ainsi, « le président commence par rappeler aux membres la mémoire des savants A. von le Coq, F. W. K. Muller et W. Barthold [tous trois disparus en $1930^{41}$; tous les membres de la section se lèvent de leurs sièges pour honorer leur mémoire » (Congrès international des orientalistes $1932: 116)$.

Se succèdent ensuite une demi-douzaine d'intervenants parmi lesquels les professeurs Kowalski de Cracovie ( Les Turcs et la langue turque en Bulgarie du nord-est ${ }^{42}$ ), Deny de Paris («Le turc était-il à l'origine une langue monosyllabique?») et Margoliouth d'Oxford (au sujet d'un manuscrit inédit sur la dynastie post-timouride des Shaybanides). Le délégué turc Reşit Saffet, membre fondateur de la Société d'histoire turque et député au parlement d'Ankara, planche pour sa part sur « la turcologie dans ses rapports avec l'orientalisme en général». Citons extensivement cette communication, qui fut probablement la toute première défense et illustration de la « thèse d'histoire » sur la scène académique internationale :

La turcologie, qui est devenue une des sections les plus vastes du groupe des études orientales [...], s'enrichit tous les jours, non seulement des progrès qui se réalisent dans l'archéologie, l'anthropologie, l'ethnographie, la linguistique, l'histoire et le folklore turcs proprement dits, mais aussi de tous les nouveaux résultats enregistrés dans les sciences pareilles ayant pour objet les peuples et les contrées du pourtour de l'Asie et de l'Europe orientale.

C'est ainsi que l'ancienne histoire et la vieille civilisation des Turcs d'avant Jésus Christ se sont trouvées considérablement éclairées à l'Est, par les très précieux travaux du professeur Pelliot ${ }^{43}$, comme des horizons leur avaient été ouverts bien avant par les études akkadiennes et sumériennes de Lenormand, qui ne sont pourtant ni l'un ni l'autre des turcologues proprement dits. [...]

De même, l'étude impartiale des civilisations et des antiquités scythiques, karpathiques et khazares, dont on possède un si abondant matériel [...], pourraient, nous semble-t-il, contribuer puissamment à celle de la civilisation hunnique.

Les inscriptions runiques que j'ai trouvées au fond du Segdan-fjord en Norvège, les Eddas islandais, le style architectural des églises en bois que j'ai vues au pays des Houtzoules dans les Tatras, les souvenirs évoqués par les noms de lieux comme Hunneschans en Hollande, jusqu'à l'histoire de St. Gall en Suisse et des Bigoudins de Bretagne au bord de l'Atlantique, méritent d'être étudiés à notre point de vue spécial comme pouvant servir, ne fût-ce que dans une faible mesure, à l'explication de beaucoup de problèmes demeurés obscurs, encore que les éléments de certitude nous fassent défaut pour le moment.

Tout ceci nous montre que la turcologie, bien que fondée et fortifiée par des savants de génie tels que Radloff, Thomsen et autres, n'est pas encore entrée dans la phase 
définitive d'organisation scientifique que son ampleur et sa maturité méritent déjà à tous égards.

Du triple point de vue de l'ethnographie, de la langue et de la civilisation, l'histoire des Turcs et de leur civilisation au moins trois fois millénaire reste donc à être objectivement reconstituée dans son ensemble, en ramenant à leur juste mesure les rôles des influences religieuses chrétienne et musulmane d'une part, et des tendances politiques et dynastiques de l'autre.

C'est la tâche à laquelle s'est courageusement vouée l'« Association pour l'Etude de l'histoire des Turcs » [c'est-à-dire la Société d'histoire turque] placée sous le haut patronage de Gazi Moustafa Kemal, son initiateur, tâche lourde et de longue haleine s'il en fut, à la réalisation de laquelle nous convions respectueusement les éminents maîtres de l'Orientalisme contemporain et particulièrement les illustres professeurs européens et américains qui honorent la turcologie actuelle ([Atabinen] 1932).

Entre les «études turques " des uns (Européens, Américains) et la "science turque " des autres (Turcs), la frontière apparaît au premier abord poreuse. Mais il faut lire ce long extrait à l'aune du millénarisme en vogue à Ankara. Avec la conviction d'un missionnaire de la science, Reşit Bey nous parle de la turcologie qui vient plutôt que de «l'actuelle »: de celle que la « réforme de l'Histoire » menée sous la houlette du Gazi dont on sait l'investissement personnel sur le sujet - a mise à l'ordre du jour. Cette Zukunftsturkologie, si l'on nous passe l'expression, est le dépassement de la turcologie des Européens. Elle est porteuse de la révolution scientifique plus large que la « Turquie nouvelle » et ses intellectuels ont vocation à apporter au monde. Elle embrassera bien sûr les Scythes, les Huns et les Khazars, clientèle habituelle des «turqueries conventionnelles »; elle promènera surtout son regard, à travers trois millénaires, de la Chine à la Mésopotamie, puis jusqu'à la Bretagne et la Scandinavie, sans négliger la lointaine Islande; elle conquerra enfin les Amériques, comme en témoignent encore des publications "linguistiques" et "ethnographiques" récentes sur les relations protohistoriques entre « Turcs et Peaux-Rouges » (Türkkan 1999) ou sur les similitudes somatiques et linguistiques qui solidarisent « les Mayas et la turcité » (Doğan 2007). 

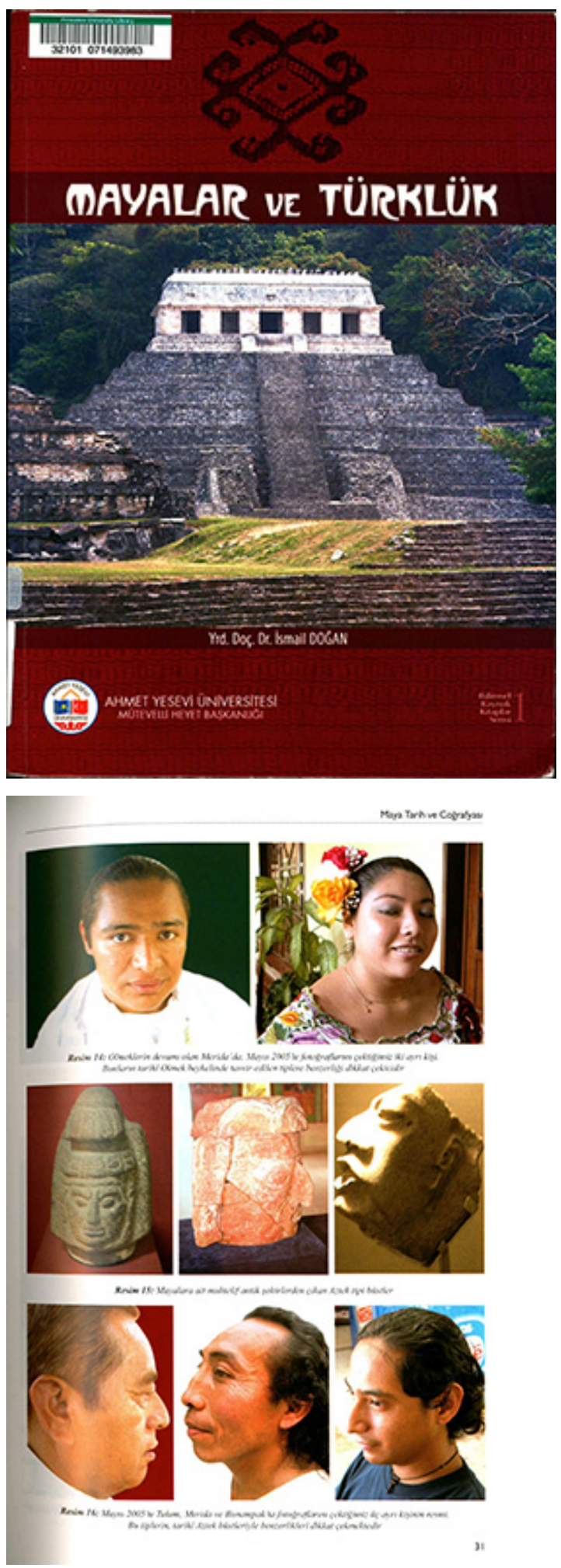

Fig. 5. Couverture et extrait de Doğan, İsmail (2007), Mayalar ve Türklük, Ankara, Ahmet Yesevi Üniversitesi.

On ne s'est guère avisé de ce que "l'hyperturcologie » turque des années 1930 est, avant l'heure, une théorie de la globalisation culturelle. Intéressant retournement de la relation d'objectivation orientaliste, en vertu duquel la turcologie cesse d'être le nom d'un savoir à travers lequel le monde, et singulièrement l'Europe, objectivent les Turcs, 
pour être celui à l'aune duquel ces derniers, resubjectivés, reprennent connaissance et possession du monde. Et puis si la turcologie a été un temps européenne, ce n'est pas bien grave puisqu'en leurs tréfonds culturels, les Européens sont des Turcs qui s'ignorent. Ce n'est donc pas la turcologie qui est européenne mais l'Europe qui, en ses savoirs comme en ses langues, reste turque ${ }^{44}$.

\section{III. Épilogue. Pour une critique du turcologisme}

Nous avons commencé cet essai en annonçant le dénombrement d'une série d' « évidences ». Tout d'abord, il n'est guère possible de fonder sur une rationalité savante la distinction entre "turcologie » et «études turques»: non seulement la distinction n'existe pas dans toutes les langues mais même quand elle existe elle n'est guère stabilisée. S'il est bien clair que les deux désignateurs reflètent divers tropismes et vogues intellectuels, notamment en termes de focalisation géographique et d'appropriation disciplinaire, on ne saurait se proclamer quitte de l'une en se revendiquant des autres. Ensuite turcologie est un mot lâche. Il change de sens comme de chemise, dans le temps et dans l'espace, voire d'une langue et d'un individu à l'autre. Hier la turcologie pouvait désigner les seules études ottomanes («l'osmanologie»), aujourd'hui encore elle accorde, de fait sinon de droit, une place exorbitante à la Turquie contemporaine, motivant en retour certains efforts vigoureux pour rétablir plus à l'est (vers la bien mal nommée Asie centrale) le centre de gravité du « domaine », si ce n'est rejeter la Türkeikunde du côté du « journalisme ».

Cette chasse aux évidences amène à une autre conclusion : en tant que champ discursif (plutôt que disciplinaire), la turcologie transnationale est profondément segmentée par la coupure Turquie/Reste du monde. On a vu qu'une proximité pour ainsi dire congénitale lie la Turquie, comme formation stato-nationale, à la production turcologique, entendue comme gisement de ressources symboliques. Si bien qu'il est parfois difficile de faire le départ entre les expressions les plus érudites du discours nationaliste turc (le turquisme académique) et les pratiques les plus politisées de ce qu'il faut bien appeler une turcologie d'État ${ }^{45}$. Opérée dès le second XIX ${ }^{\mathrm{e}}$ siècle, la resémantisation du nom turc par le nationalisme éponyme a produit et continue de produire des effets d'interférence quotidiens. La manifestation la plus ordinaire en est peut-être la "difficulté » - certainement provisoire et sans nul doute tournable - que connaît le turc (de Turquie) à distinguer ce qui est turc de ce qui est turk. Son expression la plus extrême fut sans doute la croyance des intellectuels organiques des années 1930 (on en rencontre toutefois des expressions contemporaines) qu'ils inauguraient une ère nouvelle - et mondiale - dans l'histoire de la turcologie (notre " anthropoturcologie", à l'ombre des thèses d'histoire et de la langue-soleil). Une illustration plus "douce» de ce nationalisme turcologique est la césure que certains opèrent entre " turcologie turque " et " étrangère ». Ainsi au Dictionnaire des turcologues étrangers (Eren 1998) fait pièce une galerie de portraits intitulée Les turcologues de Turquie et ceux qui ont ceuvré en faveur de la langue turque présentée comme la contrepartie «nationale » au précédent (Gülensoy 2012). Dans le même ordre d'idée, on évoquera la pratique qui consiste à confondre, au niveau discursif (par exemple à travers l'usage du "nous ») aussi bien qu'iconographique, les répertoires de la science et de l'identité. La fréquente reproduction d'une imagerie nationaliste en couverture de publications scientifiques en est une illustration. 


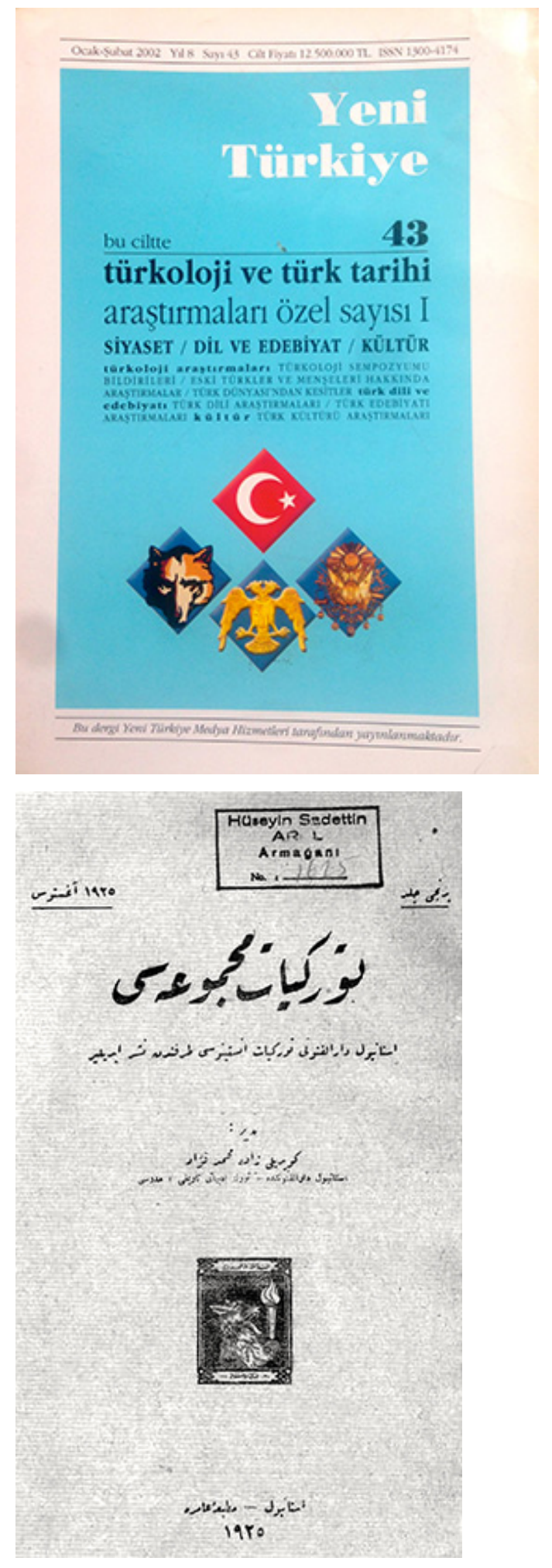




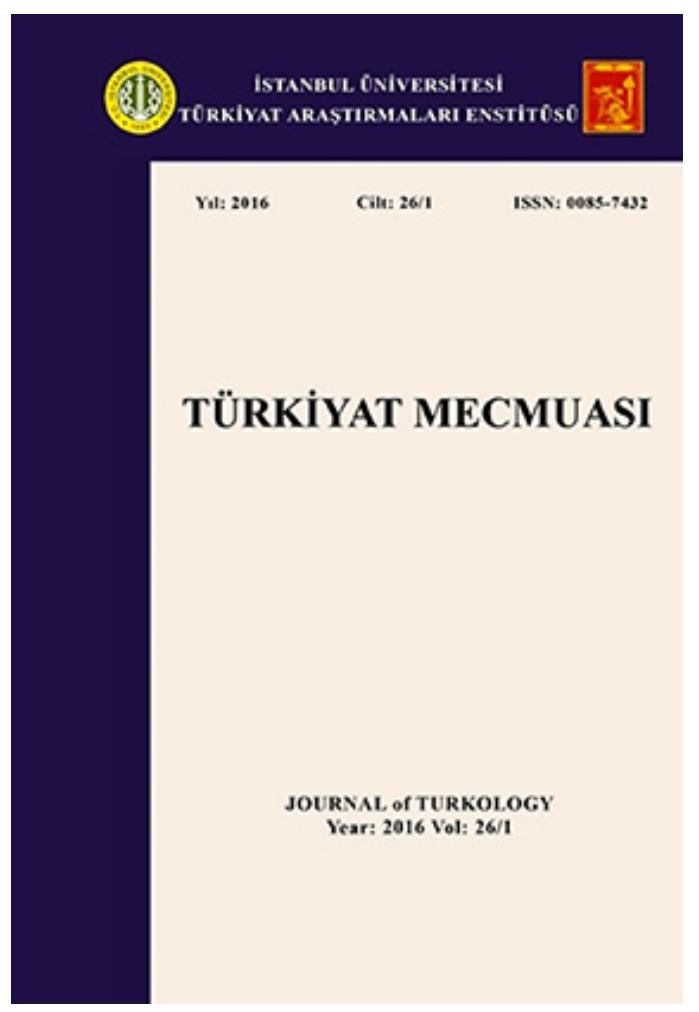

Fig. 6. Couvertures d'exemplaires des revues Yeni Türkiye (2002) et Türkiyat Mecmuası (1925 et 2016).

De la tête de loup symbolisant la période centrasiatique pré-islamique de l'«Histoire turque » au drapeau national, de l'aigle bicéphale seldjoukide aux armoiries ottomanes, on trouve, en couverture de ce numéro de la revue Yeni Türkiye intitulé « Turcologie et histoire turque » (2002), une illustration de la confusion des genres entre science et nationalisme. Il en va de même concernant la Türkiyat Mecmuasl, où il est vrai que le loup, bien visible sur le premier numéro (1925), s'est rendu plus discret (en haut à droite dans la livraison de 2016). On peut raisonnablement avancer que la récupération de cette figure totémique de l'Orkhon (Roux 1962 : 14-15) par l'extrême-droite raciste, dans les années 1930, aurait dû disqualifier l'usage de cette iconographie.

En sa qualité de «science nationale », la Türkoloji a pignon sur rue dans la totalité des facultés de lettres depuis 1982, si bien que les universités turques sont aujourd'hui les principales pourvoyeuses de savoir turcologique (Akalın 2002:13), tandis qu'ailleurs dans le monde les départements d'études turques ou de turcologie se trouvent en situation de marginalité relative (sauf peut-être en Allemagne). La turcologie de Turquie s'apparenterait même, quelquefois, à une politique publique, en ce sens qu'elle se trouve articulée à la promotion des intérêts de l'État turc sur la scène internationale. Elle a par exemple donné son nom à un programme de soft power, le Türkoloji Projesi. Lancé en 1999 sous la tutelle du conseil de l'Enseignement supérieur (YÖK) et reposant, depuis 2011, sur les instituts Yunus Emre, celui-ci consiste à déléguer de jeunes docteurs frais émoulus des universités turques auprès d'établissements partenaires, de New Delhi à Saint-Petersbourg, d'Alger à Shanghaï en passant par Zagreb, Bakou, Astana ou Sanaa. Or, ces «missionnaires de la turcologie » sont avant toute chose les représentants de leur pays et de leur langue ${ }^{46}$.

60 On peut se demander dans quelle mesure le complexe d'évidences et de confusions analysées ici fait système. Des multiples énoncés qui visent à établir l'origine, l'identité 
ou la parenté des gens et des langues d'Anatolie et d'Asie centrale aux fantasmes hyperdiffusionnistes qui inspirent la cartothèque de la Turquie républicaine, en passant par les définitions ordinaires qu'on lit sur Wikipédia, c'est bien cette quête $d u$ même qui, dans la durée, semble donner à la turcologie l'allure d'une «science de la tautologie ». Les sociologues mobilisent le mot sociologisme pour désigner les effets de surinterprétation que leurs propres paradigmes sont susceptibles d'imposer au réel et aux individus; les historiens utilisent la notion d'historicisme pour critiquer la téléologie qui consiste à produire de la causalité dans le récit des événements qu'ils enchaînent; nous proposons d'appeler turcologisme la façon dont la turcologie, comme discours scientifique hanté par un nationalisme turc à fort contenu culturaliste si ce n'est racialiste, assigne et naturalise quelquefois l'histoire et le monde : un monde dans lequel la turcité est à la fois un postulat de départ et ce qu'il s'agit d'expliquer. Il ne s'agit évidemment pas de dénoncer la chose comme une fausse science, ce qui n'aurait guère de sens, mais de s'autoriser un soupçon d'ironie, s'il nous est permis de parler ainsi, si ce n'est de suspicion, chaque fois qu'il est question de turqueries et de turcologie ${ }^{47}$.

\section{BIBLIOGRAPHIE}

Ahıska, Meltem (2010). Occidentalism in Turkey. Questions of Modernity and National Identity in Turkish Radio Broadcasting, Londres-New York, I. B. Tauris.

Meltem Ahıska (2008). « Orientalism/Occidentalism: The Impasse of Modernity », in Gürsoy Sökmen, Müge; Ertür, Başak (eds.) 2008, pp. 137-154.

Akalın, şükrü Halûk (2002). « Türkiye'de ve Dünyada Türk Dili Üzerine Çalışmalar ve Türk Dil Kurumu », Yeni Türkiye 43-46 (Türkoloji ve Türk Tarihi Araştırmaları I, II \& III »), pp. 11-17.

Aymes, Marc ; Ferry, Maroussia ; Özkoray, Hayri Gökşin (2012). « Aires culturelles et sciences sociales, ou les deux cultures : à propos du "séminaire interdisciplinaire d'études turques" », $L a$ lettre de l'EHESS 55, URL: http://lettre.ehess.fr/4461.

Aytürk, Illker (2004). « Turkish Linguists against the West: The Origins of Linguistic Nationalism in Atatürk's Turkey», Middle Eastern Studies 40 (6), pp. 1-25.

[Atabinen], Rechid Safwet Bey (1932). « La turcologie dans ses rapports avec l'orientalisme en général », Actes du XVIII e Congrès des orientalistes. Leiden, 7-12 septembre 1931, Leiden, Brill, pp. 122-124.

Babinger, Franz (1919). « Die türkischen Studien in Europa bis zum Auftreten Josef von HammerPurgstall », Die Welt des Islams 7, pp. 103-129.

Barthold, Vassili Vladimirovich (1947). La découverte de l'Asie. Histoire de l'orientalisme en Europe et en Russie, Paris, Payot (trad. Basile Nikitine).

Bazin, Louis (1953). « Notes sur les mots "Ogiuz" et "Türk" », Oriens 6 (2), pp. 315-322.

Bazin, Louis (1958). « La turcologie : bilan provisoire », Diogène 1, pp. 98-130. 
Bazin, Louis (1988). « Notes d'anthroponymie turque ancienne : l'inscription sibérienne de Barik-I (région de Touva) ", in Temimi, Abdeljelil (ed.), Mélanges Professeur Robert Mantran, Zaghouan, Publications du CEROMDE, pp. 43-50.

Berta, Årpád ; Brendemoen, Bernt ; Schönig, Claus (eds.) (1996). Symbolae turcologicae. Studies in honour of Lars Johanson on his sixtieth birthday 8 March 1996, Istanbul, Swedish Research Institute.

Beşikçi, İsmail (1991). Türk Tarih Tezi, Güneş-Dil Teorisi ve Kürt Sorunu, Ankara, Yurt Kitap Yay.

Bossaert, Marie (2016). « Connaître les Turcs et l'Empire ottoman en Italie. Construction et usages des savoirs sur l'Orient de l'Unité à la guerre italo-turque », Paris-EPHE, Florence, Scuola Normale Superiore.

Boutier, Jean ; Passeron, Jean-Claude ; Revel, Jacques (eds.) (2006). Qu'est-ce qu'une discipline?, Paris, Éditions de l'EHESS.

Brockelmann, Carl (1928). Mitteltürkischer Wortschatz nach Mahmūd al-Kā̌šarìis Dìvān lugāt at-Turk, Budapest-Leipzig, Bibliotheca Orientalis Hungarica.

Brosset, Marie-Félicité (1838). « Histoire diplomatique du patriarche arménien de Constantinople, Avédik », Bulletin scientifique publié par l'académie impériale des sciences de Saint-Pétersbourg et rédigé par son secrétaire perpétuel IV, p. 88.

Caferoğlu, Ahmet (1984). Türk Dili Tarihi I-II, Istanbul, Enderun Kitabevi (première édition 1958).

Cahen, Claude (1988). La Turquie pré-ottomane, Istanbul, Institut français d'études anatoliennes.

Cahun, Léon (1896). Introduction à l'histoire de l'Asie. Turcs et Mongols des origines à 1405, Paris, Armand Colin \& $C^{\text {ie }}$.

Çakmak, Cihan (2013). « Dîvânü Lugâti't-Türk ve Kilisli Muallim Rifat Belge », in Hasan, şener (ed.), I. Uluslararası Muallim Rifat Kilis ve Çevresi Sempozyumu (Kilis 16-17 Mayıs 2013), Kilis, Kilis 7 Aralık Üniversitesi Muallim Rıfat Eğitim Fakültesi, pp. 513-527.

CETOBAC (2017). « Dossier d'auto-évaluation », rapport quinquennal d'activité, à paraître.

Chartier, Roger (1998). « "La chimère de "l’origine". Foucault, les Lumières et la Révolution française ", Au bord de la falaise. L'histoire entre certitudes et inquiétude, Paris, Albin Michel, pp. 132-160.

Congrès international des orientalistes (1932). Actes $d u$ XVIII ${ }^{e}$ Congrès international des Orientalistes. Leiden 7-12 septembre 1931, Leiden, E. J. Brill.

Copeaux, Étienne (1997). Espaces et temps de la nation turque : analyse d'une historiographie nationaliste (1931-1993), Paris, CNRS Éditions.

Copeaux, Étienne (2000) Une vision turque du monde à travers les cartes de 1931 à nos jours, Paris, CNRS.

Dankoff, Robert (2008a). " Qaraqhanid Literature and the beginnings of Turko-Islamic culture », From Mahmud Kaşgari to Evliya Çelebi. Studies in Middle Turkic and Ottoman Literatures, Istanbul, The Isis Press, pp. 11-18.

Dankoff, Robert (2008b). « Kašğari on the tribal and kinship organization of the Turks », From Mahmud Kaşgari to Evliya Çelebi. Studies in Middle Turkic and Ottoman Literatures, Istanbul, The Isis Press, pp. 57-76.

Davids, Arthur Lumley (1832). Grammar of the Turkish Language, Londres, Parbury \& Allen. 
Davids, Arthur Lumley (1836). Grammaire turke, précédée d'un discours préliminaire sur la langue et la littérature des nations orientales, traduit par Sarah Davids, Londres, W. H. Allen.

Jean-Pierre Drège et Michel Zink (eds.) (2013). Paul Pelliot : de l'histoire à la légende. Colloque international organisé par Jean-Pierre Drège, Georges-Jean Pinault, Christina Scherrer-Schaub et PierreÉtienne Will au Collège de France et à l'Académie des Inscriptions et Belles-Lettres (Palais de l'Institut), 2-3 octobre 2008, Paris, Académie des Inscriptions et Belles-Lettres.

Deny, Jean (1921). Grammaire de la langue turque (dialecte osmanli), Paris, Leroux.

Deny, Jean (1926). « Compte rendu de : A Vahid Bey, A Condensed Dictionary English-Turkish pronouncing and explanatory and including current historical and geographical names, officially adopted by the Commissariat for Public Instruction of the Turkish Republic, by Commander A. Vahid Bey, Turkish Navy - Constantinople, 1924 (1340) (Oxford University Press, Humphrey Milford) », Journal Asiatique CCIX, pp. 129-139.

Deny, Jean (2010) ; Szurek, Emmanuel (ed.), « Rapport au recteur Charléty sur le $3^{\mathrm{e}}$ kurultay de la langue (1936) », Turcica 42, pp. 305-328.

Deny, Jean (1945). « La Turquie », conférence à l’École de guerre, Paris, 24 avril 1945 (Archives privées de Jean Deny, EHESS, Paris).

Deny, Jean (1948). « Le turc à l'École nationale des langues orientales », Cent-cinquantenaire de l'École des langues orientales, Paris, pp. 31-32.

Deny, Jean (1950). «Structure de la langue turque. Extrait des Conférences de l'Institut de linguistique de l'Université de Paris, IX, année 1949 », Paris, tiré-à-part, pp. 17-51.

Deny, Jean ; Grønbech, Kaaren ; Scheel, Helmuth ; Togan, Zeki Velidi ; Boratav, Pertev Naili (eds.) (1959). Philologiae Turcicae Fundamenta : jussu et auctoritate Unionis universae studiosorum rerum orientalium auxilio et opera Unitarum Nationum Educationis Scientiae Culturae Ordinis, una cum praestantibus Turcologis tomus primus, Aquis Mattiacis [Wiesbaden], F. Steiner.

Doğan, İsmail (2007). Mayalar ve Türklük, Ankara, Ahmet Yesevi Üniversitesi.

Eissenstat, Howard (2002). « Turkic Immigrants/Turkish Nationalism: Opportunities and Limitations of a Nationalism in Exile », The Turkish Studies Association Bulletin 25 (2)-26 (1), pp. 25-50.

Ercilasun, Ahmet (2004). Başlangıçtan Yirminci Yüzyıla Türk Dili Tarihi, Ankara, Akçag.

Eren, Hasan (1998). Türklük Bilimi Sözlüğü I. Yabancı Türkologlar, Ankara, TDK.

Ermers, Robert (1999). Arabic Grammars of Turkic. The Arabic Linguistic Model Applied to Foreign Languages \& Translation of 'Abū Hayyān al-'Andalusī's Kitāb al-'Idrak li-Lisān al-'Atrāk, Leyde, Brill.

Ersanl, Büşra (1992). İktidar ve Tarih : Türkiye'de "Resmi Tarih" Tezinin Oluşumu (1929-1937), Istanbul, Afa Yay.

Espagne, Michel (2013). « Armin Vambéry. Les pérégrinations d'un derviche Passeurs d'Orient », dans Michel Espagne et Perrine Simon-Nahum (dir.), Passeurs d'Orient. Les Juifs dans l'orientalisme, Paris, Éditions de l'Éclat, pp. 69-84.

Gal, Susan ; Irvine, Judith (1995). « The Boundaries of languages and disciplines: how ideologies construct difference », Social Research 62, pp. 967-1001.

Gauthier, Cécile (2010). « Nom, langue, identité : les errances du mot slave dans les discours de l'autre (à partir d'un corpus lexicographique - $18^{\mathrm{e}}-20^{\mathrm{e}}$ siècles - et romanesque - années 1880-1930 - de langue française et allemande) ", Slavica Occitania 30, pp. 331-340. 
Georgeon, François (1980). Aux origines du nationalisme turc. Yusuf Akçura (1876-1935), Paris, Éditions ADPF.

Georgeon, François ; Vatin, Nicolas ; Veinstein, Gilles (eds.). Avec la collaboration d'Elisabetta Borromeo (2015). Dictionnaire de l'Empire ottoman, Paris, Fayard.

Giraud, René (1960). L'Empire des Turcs célestes. Les règnes d'Elterich, Qapghan et Bilgä (680-734). Contribution à l'histoire des Turcs d'Asie centrale, Paris, Librairie d'Amérique et d'Orient AdrienMaisonneuve.

Gökalp, Ziya (1976) ; Kaplan, Mehmet (ed.). Türkçülüğün Esasları, Istanbul, Millî Eğitim Basımevi.

Gülensoy, Tuncer (2012). Türkiye Türkologları ve Türk Diline Emek Verenler (1800-1950: Türkolojinin 150 Yılı), Ankara, Akçă̆.

Gürsoy Sökmen, Müge; Ertür, Başak (eds.) (2008). Waiting for the Barbarians. A Tribute to Edward Said, Londres-New York, Verso.

Herzog, Christoph (2010). « Notes on the Development of Turkish and Oriental Studies in the German Speaking Lands », Türkiye Araştırmaları Literatür Dergisi 8 (15), pp. 7-76.

İnalcık, Halil (2002). « Hermenötik, Oryantalizm, Türkoloji », Doğu Batı 20 (1), pp. 13-39.

İnan, Abdülkadir (1936). Türkoloji Ders Hülasaları, Istanbul, Devlet Basımevi.

İnan, Abdülkadir (1936). Güneş-Dil Teorisi Üzerine Ders Notları. Türkoloji. II, Istanbul, Devlet Basımevi.

Işıksel, Güneş (2014). « Atlantisme et érudition. Dans les coulisses des Philologiae Turcicae Fundamenta (1948-1963) », in Işıksel, Güneş ; Szurek, Emmanuel (eds.), Turcs et Français. Une histoire culturelle 1860-1960, Rennes, Presses universitaires de Rennes, pp. 353-364.

İslâm Ansiklopedisi, Istanbul, Türkiye Diyanet Vakfı.

Jakobson, Roman (2003). Essais de linguistique générale. Les fondations du langage, Paris, Les Éditions de Minuit, traduction et préface de Nicolas Ruwet.

Kaplony, Andreas (2008). « Comparing Al-Kāshgarī map to his text: On the visual language, purpose and transmission of Arabic-Islamic Maps », in Forêt, Philippe ; Kaplony, Andreas (eds.), The Journey of Maps and Images on the Silk Road, Leiden, Brill, pp. 137-154.

Kazancıgil, Aykut ; Vergili, Ayhan (2013). « Millî Tetebbular Mecmuası (1331-1915): Bir Devlet Mecmuası ", in Kazancıgil, Aykut ; Vergili, Ayhan ; Hatemi, Hüsrev (eds.), Bilim Tarihi ve Kültür Yazıları, Istanbul, İşaret Yay., pp. 445-466.

Kilisli Muallim Rifat ed. (1333-1335). Mahmut bin el-Hüseyin bin Mehmet el-Kaşgarî, Kitab-i Divani Lûgat-it-Türk, Darülhilafetül Aliye [Istanbul], Matbaa-yi Amire.

Knüppel, Michael (s.d.). « Geschichte der Turkologie », URL: http://uni-goettingen.de/de/ 349075.html.

Kocaoğlu, Timur (2004). « Diwanu Lugatı't-Turk and Contemporary Linguistics », Journal of Turkic Civilization Studies 1, pp. 165-169.

Laut, Jens-Peter (2013). Was ist Turkologie? Überlegungen zu einem sogenannten Orchideenfach, Bonn, Max Weber Stiftung - Deutsche Geisteswissenschaftliche Institute im Ausland.

Laut, Jens-Peter (2015). What is Turkology? Reflections on a so-called "exotic" subject (trad. Teresa Gatesman). Bonn, Max Weber Stiftung - Deutsche Geisteswissenschaftliche Institute im Ausland. 
Lellouch, Benjamin (2013). « Qu'est-ce qu'un Turc ? (Égypte, Syrie, XVI ${ }^{\mathrm{e}}$ siècle) », European Journal of Turkish Studies, URL: http://ejts.revues.org/4758.

Maksudi, Sadri (1977). « Dostum Yusuf Akçura », Türk Kültürü 174, pp. 346-354.

Mangold, Sabine (2011). « Entre diplomatie et érudition : Josef von Hammer-Purgstall et son réseau en Europe et dans l'Empire ottoman », Basch, Sophie ; Chuvin, Pierre ; Espagne, Michel ; Seni, Nora ; Leclant, Jean (eds.), L'orientalisme, les orientalistes et l'Empire ottoman de la fin du XVIII à la fin du XX siècle. Actes du colloque international (Palais de l'Institut de France, 12 et 13 février 2010), Paris, Académie des inscriptions et belles-lettres, pp. 205-218.

Ministère des Affaires Étrangères (1926). Bulletin périodique de la presse turque. Du 7 Décembre 1925 au 13 Juin 1926, 45, pp. 1-12.

Olender, Maurice (2005). La chasse aux évidences. Sur quelques formes de racisme entre mythe et histoire, Paris, Galaade.

Peacock, Andrew Charles Spencer (2010). Early Seljūk History. A New Interpretation, Londres-New York, Routledge.

Pekaçar, Bekir Yavuz (2012). « Türkiye Türkologları ve Türk Diline Emek Verenler (1800-1950: Türkolojinin 150 Yılı) », Gazi Türkiyat Türkoloji Araştırmaları Dergisi 11, pp. 257-260.

Postel, Guillaume (1575). Des histoires orientales et principalement des Turkes ou Turchikes et Schitiques ou Tartaresques et autres qui en sont descendues, Paris, Imprimerie de H. de Marnef.

Redhouse, James William (1978). A Turkish and English Lexicon, shewing in English the significations of the Turkish terms, Istanbul, Çağrı Yay.

Ricœur, Paul (2000). La mémoire, l'histoire, l'oubli, Paris, Seuil.

Róna-Tas, András (1991). An Introduction to Turkology, Szeged, Attila József University.

[Rossi, Ettore] (1933). « Conferenza di Ettore Rossi sugli studi di Turcologia in Italia », Oriente Moderno 10, pp. 511.

Roux, Jean-Paul (1962). « La religion des Turcs de l'Orkhon, des VII ${ }^{\mathrm{e}}$ et VIII ${ }^{\mathrm{e}}$ siècles (premier article) », Revue de l'histoire des religions 161 (1), pp. 1-24.

Şemsettin Sami (2010). Kamus-ı Türkî, Yavuzarslan, Paşa (ed.), Ankara, TDK.

Siblot, Paul (1999). « Appeler les choses par leur nom. Problématiques du nom, de la nomination et des renominations ", Salih Akin (dir.), Noms et re-noms. La dénomination des personnes, des populations, des langues et des territoires, Rouen, Publications de l'Université de Rouen, pp. 13-31.

Süleyman Hüsnü Paşa (2006). Toparlı, Recep et Yücel, Dilek (eds.), İlm-i Sarf-ı Türkî, Ankara, TDK. Szurek, Emmanuel (2013). « Gouverner par les mots. Une histoire linguistique de la Turquie nationaliste ", thèse de doctorat non publiée, Paris, EHESS.

Szurek, Emmanuel (2014). « Les Langues orientales, Jean Deny, les Turks et la Turquie nouvelle. Une histoire croisée de la turcologie française (XIX ${ }^{\mathrm{e}}-\mathrm{XX}^{\mathrm{e}}$ siècle) », in Işıksel, Güneş ; Szurek, Emmanuel (eds.), Turcs et Français. Une histoire culturelle 1860-1960, Rennes, Presses universitaires de Rennes, pp. 327-352.

Szurek, Emmanuel (2017). « Connaissez-vous la théorie de la langue-soleil ? Une histoire européenne du fantasme scientifique dans la Turquie des années 1930 ", in Couto, Déjanirah ; Péquignot, Stéphane (eds.), Les langues de la négociation. Approches historiennes, Rennes, Presses universitaires de Rennes, pp. 235-270. 
Tankut, Hasan Reşit (1938). « Le mot "Alp" et le foyer de la race alpine », Türk Dili 27-28, pp. 149-165.

Tekin, Talat ; Ölmez, Mehmet (1995) Türk Dilleri = Les Langues turques, Ankara, T.C. Kültür Bakanlı̆̆.

Temir, Ahmet (1991). Türkoloji Tarihinde Wilhelm Radloff Devri. Hayatı, İlmî Kişiliği, Eserleri, Ankara, TDK.

Togan, Zeki Velidi (1999). Hâtıralar, Istanbul, Diyanet Vakfı Yay.

Tulga, Yiğit (2007). « Turkic (Türkik) diller demesek, ne desek? », Turkcesivarken.com, URL : http://turkcesivarken.com/yazismalik/viewtopic.php?f=2\&t=463\&p=1090.\%20

Tülücü, Süleyman (2000). « Prof. Abdülkadir İnan Üzerine Bazı Notlar », Atatürk Üniversitesi Türkiyat Araştırmaları Enstitüsü Dergisi 15, pp. 201-211.

Türk Dil Kurumu, Güncel Türkçe Sözlük, URL : http://www.tdk.gov.tr/index.php?option=com_gts.

Türkkan, Reha Oğuz (1999). Kızılderililer ve Türkler, Istanbul, E Yay.

[Uşaklıgil], Halid Ziya (1999). Türkay, Kaya (ed.), Kavẩid-i Lisân-ı Türkî, Ankara, TDK.

Valensi, Lucette (1972). « Le mot "nègre" dans les dictionnaires d'Ancien régime; histoire et lexicographie », Langue Française 15, pp. 79-104.

Vatin, Nicolas (2001). Conférence d'ouverture de M. Nicolas Vatin, directeur d'études, Études ottomanes, $X V^{e}$-XVIII ${ }^{e}$ siècle : 10 nov. 2000, Paris, École pratique des hautes études.

Veinstein, Gilles (2000). Leçon inaugurale : faite le vendredi 3 décembre 1999, Collège de France, Chaire d'histoire turque et ottomane, Paris, Collège de France.

Veinstein, Gilles (2014). « La turcologie avant Jean Deny. La place des drogmans », in Işıksel, Güneş; Szurek, Emmanuel (eds.), Turcs et Français. Une histoire culturelle 1860-1960, Rennes, Presses universitaires de Rennes, pp. 309-316.

Veinstein, Gilles ; Nicolas, Michèle (eds.) (1994). Les Turcs, des mots, des hommes (préf. J. Hamilton), Budapest, Akadémiai Kiadó.

Zürcher, Erik Jan (2014). « Monologue to Conversation: Comparative Approaches in Turkish Historiography », Turkish Studies 15 (4), pp. 589-599.

\section{NOTES}

1. Nous remercions vivement les deux relecteurs anonymes de l'EJTS ainsi que Marc Aymes pour leurs précieuses remarques. Notre réflexion a également bénéficié de nombreux échanges avec Marie Bossaert depuis la gestation de ce dossier (cf. l'introduction, dont la présente contribution emboîte le pas). Cet essai est aussi le fruit d'un compagnonnage de longue haleine avec les chercheurs anciens et actuels du CETOBAC (ci-devant «Centre d'histoire du domaine turc ») de l'EHESS. Enfin il déplie et approfondit des réflexions esquissées dans Szurek 2014. Ajoutons que, centré sur Paris, l'Europe occidentale et la Turquie républicaine, ce texte procède d'une trajectoire et d'une subjectivité scientifiques qui nous sont propres. Il va de soi que d'autres facettes de la «relation turcologique " se dévoileraient à partir d'autres terrains d'observation (Russie, mondes arabe, persan, indien ou encore chinois). Merci pour finir à Augustin Jomier qui nous a aidé à comprendre diverses références en allemand. 
2. Le terme de discipline est ici entendu comme « un principe de spécialisation de la recherche qui se veut à la fois logique, par sa référence à une théorie unifiée de l'intelligibilité, et fonctionnel, par ses principes d'organisation de la diversité des connaissances " (Jean Boutier et al. 2006: 7).

3. Sur l'articulation entre " aréal » et " général » (ou encore " empirique » et " théorique ») dans les études turques, cf. Marc Aymes et al. 2012.

4. Plusieurs contributions au présent dossier ont par exemple fait l'objet de présentations orales $\mathrm{au}$ « Turkologentag » qui s'est réuni à Hambourg en septembre 2016.

5. Cf. par exemple les fameux Philologiae Turcicae Fundamenta dont le titre latin traduit bien l'horizon encyclopédique, et sert de prétexte à une série de néologismes façon Médecin malgré lui, tel le pédant Turcologus et l'imaginatif Unitarum Nationum Educationis Scientiae Culturae Ordinis (l'UNESCO ayant chapeauté l'opération). Dans les années 1970, la revue française Turcica (créée en 1969) publie régulièrement des états de l'art sur les "travaux turcologiques » à travers le monde (Angleterre, URSS, Israël, Japon, Bulgarie, Scandinavie etc.) : autant de témoins de la dissémination de la « discipline ». Voir les références sur le site du département d'études turques de l'université de Strasbourg (URL: http://turcologie.u-strasbg.fr/Turcica\%20-sommaire1-30.pdf. Sur les Fundamenta, Işıksel 2014).

6. Cf. par exemple les premiers mots de Zürcher 2014 : "During my graduate studies in Turkish Studies, or rather in what at the time was still generally called "Turcology" in Leiden University in the 1970s, the idea of comparing the Ottoman Empire to other imperial constellations, or for that matter, of comparing Kemalist Turkey to other European states of the interbellum was not on our radar screen. »

7. La notion de Türkeikunde, comme sous-branche de la turcologie, est emprunté à Horst Wilfrid Brands (Laut 2013: 27) ; celle de Gesamtturkologie au spécialiste de l'ouzbek et du tchouvache Johannes Benzing (Laut $2013: 16,29$ ). Benzing la forgea en 1953, du temps où il faisait oublier à Paris les années qu'il avait passées à Berlin au service du III ${ }^{\mathrm{e}}$ Reich. Il participa notamment aux activités du Centre d'études turques de la Sorbonne (voir l'affiche, infra). (Sur Benzing membre du NSDAP puis employé du Quai d'Orsay, Herzog $2010: 50$ ).

8. À ne pas confondre avec la revue Turkish Studies. International Periodical for the Languages, Literature and History of Turkish or Turkic, turcophone malgré son titre, créée en 2006 à Ankara. URL: www.turkishstudies.net.

9. http://www.tandfonline.com/action/journalinformation? show=aimsScope\&journalCode=ftur20.

10. Cf. encore la présentation de la revue dans CETOBAC 2017 : «[l'EJTS] offre une approche par les sciences sociales et des dossiers thématiques portant surtout sur la Turquie contemporaine $[\ldots] »$.

11. Pour une réflexion approchante, cf. Herzog $2010: 7$.

12. Son modèle, que faute de sources il regrette de ne pouvoir imiter, est la Geschichte der SanskritPhilologie und indischen Altertumskunde d'Ernst Windisch (Strasbourg, K. J. Trübner, 1917-1920, 3 vols).

13. Sur Hammer comme figure de transition dans la professionnalisation de l'orientalisme viennois, cf. Mangold 2011.

14. Voir la contribution de Despina Magkanari au présent dossier.

15. Nous préparons, dans le prolongement de cette contribution, un article sur le Centre d'études turques de l'université de Paris (1935-1960), qui fut la " première » arène dévolue à la turcologie en France et à plusieurs égards «l'ancêtre» de l'actuel Centre d'études turques, ottomanes, balkaniques et centrasiatiques de l'EHESS.

16. Imaginerait-on un historien français de l'Ancien Régime se revendiquer des French studies, autrement que pour se vendre sur le marché académique du monde anglophone?

17. Pour aller plus loin, une « liste des revues académiques de turcologie » est disponible sur le site d'İsa Sari, maître de conférences à l'université Hittite de Çorum qui se présente comme un 
spécialiste de «turcologie digitale » (bilgisayarlı Türkoloji). URL: http://www.isa-sari.com/etiket/ bilgisayarli-turkoloji.

18. La distinction existe en anglais, néerlandais, espagnol, russe et indonésien (mais, curieusement, pas en allemand). En anglais elle existe au niveau adjectival (Turkic) mais non nominal (Turk dans les deux cas).

19. Cet usage souffre des exceptions. Par exemple on continue d'appeler turcs les turcophones de l'aire post-ottomane (« Turcs de Bulgarie », « Turcs chypriotes », « Turcs de Macédoine »), peutêtre en vertu d'une survivance du temps où l'Empire ottoman constituait une entité propre dont les habitants musulmans étaient indistinctement appelés « Turcs » par les Européens. Je remercie Nathalie Clayer pour ses éclaircissements.

20. Les termes «turquien ", « turc de Turquie » et «oğuz occidental » y sont proposés comme équivalents.

21. La parenté du turk et du sumérien est une hypothèse banale en Turquie. En toute rigueur, nous serions bien incapables d'en juger et nous en remettons à l'autorité d'une communauté scientifique qui, depuis les années 1930, la juge généralement avec une certaine sévérité (Deny 2010).

22. URL: https://en.wikipedia.org/wiki/Turkology.

23. Timur Kocaoğlu est membre du Türk Kültürünü Araştırma Enstitüsü d'Ankara (dont son propre père, Osman Kocaoğlu, éphémère président de la République populaire soviétique de Boukhara) avait été, en 1961, l'un des membres fondateurs. Sur le TKAE et sa place dans l'histoire de la turcologie turque, cf. la contribution d'ilker Aytürk dans ce dossier.

24. Sur les catégories collectives ("peuple», "tribu», turk») chez Mahmoud de Kashgar, cf. Dankoff 2008b ; sur la grammaire de l'appartenance dans le monde seldjoukide, Peacock 2010 (en particulier le chapitre $4:$ " The tribes and the Seljūk dynasty », pp. 47-71).

25. İA, « Dîvânü Lugati't-Türk » (Mustafa S. Kaçalin).

26. Cette carte est reproduite en couverture de l'ouvrage d'hommage à Lars Johanson : Berta et al. 1996.

27. Un récit passionnant de la redécouverte du manuscrit dans Çakmak 2013.

28. «[...] tant il est vrai que dans un Empire qui prétend toujours officiellement à la multinationalité, la focalisation sur la langue et la culture turques continue à paraître déplacée » (François Georgeon dans Georgeon, Vatin et Veinstein 2015, article « turcologie »).

29. Martin Hartmann, "Divan-1 lügat-i türk'e ait birkaç mülâhaza », Millî Tetebbular Mecmuası, II-4, pp. 166-172, cité dans Kazancigil et Vergili 2013 : 457. Des élements sur Hartmann dans Herzog $2010: 22$.

30. Cf. İA, « Brockelmann, Carl (1868-1956) » (Nuri Yüce).

31. Pour une bibliographie complète, cf. İA, « Dîvânü Lugati't-Türk », art. cit.

32. Au début de 1924, Zeki Velidi Togan séjourne à Paris où il fréquente les milieux intellectuels orientaux et orientalistes. Dans ses mémoires, il se fait notamment l'écho de l'intérêt que nourrit à cette époque Deny pour Kachgari (Togan 1999 : 465).

33. Cette publication s'appuie sur les traductions antérieures mais non publiées de Kilisli Rifat [Bilge], Abdullah Atıf [Tüzüner] et Abdullah Sabri [Karter]. İA, « Kilisli Rifat Bilge » (Ömer Faruk Akün).

34. Merci à Avi Mizrahi qui m'a signalé ce document. Sur l'histoire de la turcologie italienne, cf. Bossaert 2016.

35. Publiée en anglais (Davids 1832) et traduite en français (Davids 1836), cette grammaire fut offerte à Mahmoud II et non à Sélim III (assassiné en 1808).

36. Sur de Guignes et ses inspirateurs, voir la contribution de Despina Magkanari dans ce dossier. 
37. Ce titre interpelle: qui l'auteur identifie-t-il comme étrangers? Ceux qui n'ont pas de passeport turc? qui ne sont pas turcophones de naissance? qui n'ont pas d'«ascendance» turque?

38. Voir le sommaire de ce premier numéro sur le site de l'actuelle Türkiyat Mecmuast-Journal of Turkology de l'université d'Istanbul. URL: http://www.journals.istanbul.edu.tr/iuturkiyat/issue/ view/1023000139/showToc

39. Lequel fut le remplaçant de Jean Deny à la chaire de turc de l'École des langues orientales de Paris pendant que ce dernier accomplissait une mission à la bibliothèque khédiviale du Caire (1926-1930).

40. À l'inverse l'assyriologie et l'égyptologie disposent de leurs propres sections.

41. 1930 : annus horribilis pour la turcologie qui voit encore périr Joseph Marquart.

42. Le compte-rendu de la discussion qui suit la communication de Tadeusz Kowalski illustre la performance chez les savants réunis de rites d'appartenance à une communauté scientifique : «M. DENY constate qu'il y a encore beaucoup a faire dans les Balkans pour la turcologie. RECHID SAFWET BEY parle de l'origine des Gagaouzes qui, selon lui, est encore obscure. M. MINORSKY attire l'attention sur le fait que les habitants du Déli Ormane étaient en grande partie des Chrétiens et que le changement ei>î dans leur dialecte pouvait être comparé avec celui constaté dans le dialecte turcoman parlé en Perse. » (Congrès international des orientalistes 1932 : 118).

43. On se figure mal ce qui, dans l'œuvre de Paul Pelliot, est susceptible de renseigner l'histoire des Turks avant Jésus-Christ. Sur ce dernier, Drège et Zink 2013.

44. À lire le procès-verbal de la séance, il semble que l'assistance préféra botter en touche: «Dans la discussion MM. DENY, KOWALSKI et MINORSKY attirent 1'attention sur la difficulté qu'il y a dans l'étude de l'histoire d'un peuple nomade ». (Congrès international des orientalistes $1932: 124)$.

45. Sur la genèse de ce turquisme académique, production intermédiaire entre sciences humaines et nationalisme culturel, nous nous permettons de renvoyer à Szurek 2013 : 388 sqq.

46. La liste des postes ouverts en 2014-2015 est par exemple consultable sur le site du YÖK. URL: http://www.yok.gov.tr/web/guest/anasayfa/-/asset_publisher/64ZMbZPZlsI4/content/ turkoloji-projesi-kapsam\%C4\%B1nda-yurt-d\%C4\%B1s\%C4\%B1ndaki-universitelerdecal\%C4\%B1smak-uzere-ogretim-elemanlar\%C4\%B1-gorevlendirilecektir.

47. En clin d'œil à Étienne Copeaux, dont le blog scientifique et militant s'est longtemps appelé « Un pas de côté dans les études turques ».

\section{RÉSUMÉS}

Le mot turcologie désigne des pratiques à ce point diversifiées qu'il est douteux qu'on puisse les référer à une architecture épistémologique commune. À l'inverse, une multiplicité de notions (turcologie, études turques, "science de la turcité », "étude de la Turquie »), plus ou moins traduisibles ou interchangeables d'un pays à l'autre, sont disponibles sur le marché linguistique international. Dans cet essai, notre propos est d'approfondir la question des interférences entre deux entités interdépendantes : la Turquie, entendue comme construction nationale et comme formation étatique, et la turcologie, conçue comme modalité théorique et pratique d'organisation du travail scientifique. Deux pistes seront suivies : l'une, philologique, consistera à décortiquer les mots et les noms de la turcologie dans leur variabilité temporelle et spatiale; l'autre, 
historiographique, visera à mieux identifier les différents tropismes (nativisme, eurocentrisme, millénarisme) qui inspirent la façon dont on a écrit l'histoire des études turques au XX $\mathrm{X}^{\mathrm{e}}$ siècle.

The term Turcology refers to practices that are so diversified that it is doubtful whether they can be referred to via a single epistemological architecture. Conversely, a variety of scholarly designations ('Turkology', 'Turkic studies', 'Turkish studies', 'science of Turkishness', 'study of Turkey') are available on the international linguistic marketplace to denote this body of studies. In this essay, we intend to explore the question of tension between Turkey, understood as a national construction and a state formation, and Turcology, conceived as a theoretical and practical modality of scholarly investigation. The article will shed light on two matters: the first, philological, will consist in dissecting a few core concepts and names of Turcology in their temporal and spatial variability; the second, historiographical, will outline how the history of the field is written and why the different patterns that shape its narrative (nativism, Eurocentrism, millennialism) tell more about the so-called Turcologists than about Turcology itself.

INDEX

Keywords : Turkology, Orientalism, Philology, Historiography, Epistemology

Mots-clés : Turcologie, Orientalisme, Philologie, Historiographie, Épistémologie

\section{AUTEUR}

EMMANUEL SZUREK

Maître de conférences EHESS CETOBaC emmanuel.szurek@gmail.com 